\title{
LQR Based Optimal Topology of Hybrid-Weighted Multiagent Systems
}

\author{
Shuai Liu $\mathbb{D}^{1},{ }^{1}$ Zhijian Ji $\mathbb{D}^{1},{ }^{1}$ Haisheng Yu $\mathbb{D}^{1},{ }^{1}$ and Ting Hou $\mathbb{D}^{2}$ \\ ${ }^{1}$ School of Automation, Qingdao University, Qingdao, Shandong 266071, China \\ ${ }^{2}$ College of Mathematics and Systems Science, Shandong University of Science and Technology, Qingdao, Shandong 266590, China \\ Correspondence should be addressed to Zhijian Ji; jizhijian@pku.org.cn
}

Received 16 July 2018; Accepted 3 October 2018; Published 17 October 2018

Academic Editor: Libor Pekař

Copyright (c) 2018 Shuai Liu et al. This is an open access article distributed under the Creative Commons Attribution License, which permits unrestricted use, distribution, and reproduction in any medium, provided the original work is properly cited.

\begin{abstract}
In this paper, the optimal topology structure is studied for hybrid-weighted leader-follower multiagent systems (MASs). The results are developed by taking advantage of linear quadratic regulator (LQR) theory. We show that the multiagent star composite structure is the optimal topology which can enable the MAS to achieve the bipartite consensus. In particular, we prove that the optimal topology corresponding to the multiagent system with the first-order static leader and the second-order dynamic leader is, respectively, a hybrid-weighted star composite structure and an unevenly hybrid-weighted star composite structure. The results of the paper indicate that, in addition to the necessary information communication between leader and followers, the information exchange among followers increases the control cost of the system.
\end{abstract}

\section{Introduction}

Real-life systems related to bionics, including the animal world, ants and geese formations, can often be studied under the framework of multiagent systems. Many other practical systems have also benefited from the study of multiagent systems, such as UAV formation, motion sensor control, and team handling robots [1-32]. Consensus is one of the most extensive and in-depth studies in the research field of multiagent systems. Vicsek et al. pioneered the investigation of consensus problem [33]. The consensus problem means a group of agents which eventually converge to a common state in the exchange of information and communication among neighbors. Since the communications between agents have both positive and negative weights, C. Altanfini put forward the definition and derived determination conditions for the bipartite consensus of multiagent systems [34]. The bipartite consensus means that a set of agents finally converge to the same modulus with opposite state under the exchange of neighbor information. Based on [33], $\mathrm{Hu}$ et al. weakened the conditions into spanning trees for achieving the bipartite consensus of first-order multiagent systems [35, 36]. Along with the in-depth study of consensus problem, more and more scholars have devoted themselves to the investigation of optimization problems, in which the optimal cost control problem got special attention. Cao and Ren proposed an optimal control protocol for MASs by taking advantage of LQR theory without considering any leader. They proved that the optimal structure can be obtained in the corresponding directed graph [37]. In [38], the optimal topology structure was proposed based on the LQR theory for the first- and second-order leader-follower multiagent system. In this case, consensus is still achieved.

Armed with the forementioned results, we discuss the optimal control structure for hybrid-weighted multiagent systems. Using the LQR theory, we show that the optimal topological structure associated with the MAS of static leaders is a hybrid-weighted star composite topology; and the corresponding optimal topology structure of dynamic leader MASs is the unevenly hybrid-weighted star composite topology. Each of these two structures is proved to be the optimal solution to the bipartite consensus for the corresponding multiagent system.

The rest of this paper is organized as follows. Section 2 contains the basic knowledge of graph theory and LQR theory. In Sections 3 and 4, the optimal control structure 
problem is established and solved, for the first- and secondorder leader-follower multiagent system with hybrid weights, respectively. For the general leader-follower multiagent system, the results on optimal topology structure are reported in Section 5. In Section 6, several simulation results are analyzed. Section 7 concludes this paper.

In this paper, the following symbols are used: $\mathbb{R}$ denotes a real number set. $\mathbb{R}^{n \times m}$ represents an $n \times m$ real matrix set. Column vector $\mathbf{1}_{\mathbf{n}}$ ( or $\mathbf{0}_{\mathbf{n}}$ ) indicates that all items in each column are equal to 1 (or 0 ). $\operatorname{diag}\left\{A_{1}, \cdots, A_{n}\right\}$ is a diagonal block matrix whose diagonal elements are matrices $A_{i}, i=$ $1, \cdots, n . B=A^{1 / 2}($ or $B=\sqrt{A})$ when $B$ satisfies $B^{2}=A . A \otimes B$ represents the Kronecker product of $A$ and $B . \mathscr{I}_{n}=\{1, \cdots, n\}$ represents the index set.

\section{Preliminaries}

Graph theory can abstract the information interaction and relative relation between agents, which plays an important role in the research of distributed coordination. $G=$ $\{\mathscr{V}, \mathscr{E}, \mathscr{A}\}$ describes a hybrid-weighted directed graph. The hybrid weight means that the edge weight can take positive or negative values, with positive value indicating a cooperative relationship between two nodes, while negative value indicating a competitive relationship. $\mathscr{V}$ is the set of vertices, and $\mathscr{E}$ is the edge set. $\mathscr{A}$ is an adjacency matrix. The corresponding concrete expression is $\mathscr{V}=\{1,2, \cdots, n\}, \mathscr{E}=\{(i, j) \in \mathscr{V} \times \mathscr{V}\}$, $\mathscr{A}=\left[a_{i j}\right] \in \mathbb{R}^{n \times n}$. Edge set $\mathscr{E}$ is an abstraction of the information exchange. $(j, i) \in \mathscr{E}$ means that agent $i$ receives information from agent $j . i \in \mathcal{N}_{n}$ denotes a set with $n$ agents; and $a_{i i}=0$ means that we do not consider the self-loop. We assume that $a_{i j} a_{j i} \geq 0 . a_{i j}=0$ indicates that there is no connection between $v_{j}$ and $v_{i}$. If $v_{j}$ and $v_{i}$ are cooperative, then $a_{i j}>0$; otherwise $a_{i j}<0$. The spanning tree of a connected graph with $n$ nodes is a minimally connected subgraph of the original graph which contains all the $n$ nodes in the original graph and keeps the least connected edge of the graph.

The neighbor set of agent $i$ is denoted by $\mathscr{C}_{i}, \mathscr{C}_{i}=\{j$ : $\left.a_{i j} \neq 0\right\}$. Degree matrix $\mathscr{D}$ is a diagonal matrix with diagonal elements $d_{i i}=\sum_{j \in \mathscr{C}_{i}} a_{i j}$. Laplacian matrix $\mathscr{L}=\mathscr{D}-\mathscr{A}$. In a leader-follower multiagent system, leaders cannot receive neighbor information from follower nodes. The followers usually have at least one neighbor node. For a leaderfollower multiagent system, the star topology is a specific interconnection structure, in which all nodes are individually connected to a central hub. With the central node playing the single leader role (the central hub of the star composite structure plays the same role with respect to multiple leaders), the leader does not receive any information from other nodes while any other nodes can use the central node as a neighbor (the leader nodes cannot receive neighbor information but can send neighbor information to other nodes). In this paper, the leader is denoted by $N^{*}$ and the follower's neighbor set is denoted by $N_{i}, i \in \mathcal{N}_{n}$. The information exchange associated with the first-order multiagent system involves only position information, which is described by $G(\mathscr{A})$. The corresponding Laplacian is $L^{\mathscr{A}}$. The information exchange associated with the second-order multiagent system includes both position and velocity information, which is described by an additional digraph $G(\mathscr{V})$ and a Laplacian matrix $L^{\mathscr{V}}$. In association with the second-order multiagent system, the directed graph $G(\mathscr{A})$ represents the position information and the directed graph $G(\mathscr{V})$ represents the velocity information. These two graphs have the same structure with different edge weights. The structural model of the system is called unevenly weighted interaction graph.

2.1. Infinite-Time Linear Quadratic Regulator Theory. Consider a linear system

$$
\dot{X}(t)=G X(t)+H U(t),
$$

where $X(t) \in \mathbb{R}^{n}, U(t) \in \mathbb{R}^{m}, G \in \mathbb{R}^{n \times n}$, and $H \in \mathbb{R}^{n \times m}$. Let $Q \in \mathbb{R}^{n \times n}, R \in \mathbb{R}^{m \times m}$, which are symmetric, nonnegative, and positive definite, respectively. The following cost function is defined by

$$
\begin{aligned}
& J(U(.), X(0)) \\
& \quad=\int_{0}^{\infty}\left[X^{T}(t) Q X(t)+U^{T}(t) R U(t)\right] d t .
\end{aligned}
$$

The cost function represents the total cost of system (1) from the initial state $X(0)$ to the equilibrium state. The task of the optimal control problem is to find the minimum cost function $J(U(),. X(0))$ and the optimal control $U^{*}(t)=$ $-K^{*} X(t)$, where $K^{*}$ is called the optimal feedback gain matrix [39]. In other words, optimal control can bring the state to equilibrium without putting too much cost on it. The LQR optimal control lemma is given below.

Lemma 1 (see [39]). Suppose that system (1) is completely controllable or completely stabilizable. The algebraic Riccati equation $(A R E)$

$$
G^{T} P+P G+Q-P H R^{-1} H^{T} P=0
$$

has a unique positive-definite solution. Furthermore, the optimal control $U^{*}(t)=-R^{-1} H^{T} P X(t)$ minimizes the cost function $J(U(),. X(0))$ and makes the system asymptotically stable.

\section{LQR Based Optimal Topology of First-Order Hybrid-Weighted Multiagent Systems}

3.1. Single Leader. The first-order leader-follower multiagent system is defined as

$$
\begin{aligned}
\dot{x}_{i}(t) & =u_{i}(t), \quad i \in \mathcal{N}_{n}, \\
\dot{x}_{N^{*}}(t) & =0
\end{aligned}
$$

where $x_{i}(t) \in \mathbb{R}$ and $u_{i}(t) \in \mathbb{R}$ represent the $i$ th follower's position information and the control input information, respectively. Let $x_{N^{*}}(t)$ represent the leader's position information and denote $X(t)=\left(x_{1}(t), \cdots, x_{n}(t)\right)^{T}, U(t)=\left(u_{1}(t)\right.$, $\left.\cdots, u_{n}(t)\right)^{T}$. Then achieving consensus is to design neighboring information based feedback $u_{i}(t)\left(i \in \mathcal{N}_{n}\right)$ so that the final 
states of both the leader and followers converge to the same value. The corresponding expression is as follows

$$
\lim _{\mathrm{t} \longrightarrow \infty}\left|x_{i}(t)-x_{N^{*}}(t)\right|=0, \quad i \in \mathcal{N}_{n} .
$$

We have

$$
L_{f f}^{\mathscr{A}}=\Delta_{f f}-\mathscr{A}_{f f}
$$

where

$\Delta_{f f}$

$$
=\operatorname{diag}\left\{\sum_{j=1, i \neq j}^{n}\left|a_{1 j}\right|+\left|o_{1}\right|, \cdots, \sum_{j=1, i \neq j}^{n}\left|a_{n j}\right|+\left|o_{n}\right|\right\},
$$

$\mathscr{A}_{f f}=\left[a_{i j}\right] \in \mathbb{R}^{n \times n}$. Define the Laplacian matrix $L^{\mathscr{A}}$ as

$$
L^{\mathscr{A}}=\left(\begin{array}{cc}
L_{f f}^{\mathscr{A}} & -\alpha \\
\mathbf{0}_{n}^{T} & 0
\end{array}\right)
$$

where $L_{f f}^{\mathscr{A}} \in \mathbb{R}^{n \times n}, \alpha \in \mathbb{R}^{n \times 1}, \alpha=\left(o_{1}, \cdots, o_{n}\right)^{T}$, and $o_{i}$ refers to the connection between the leader and the $i$ th follower.

Consider the following linear protocol

$$
\begin{aligned}
u_{i}(t)= & -\sum_{j=1}^{n}\left|a_{i j}\right|\left[x_{i}(t)-\operatorname{sgn}\left(a_{i j}\right) x_{j}(t)\right] \\
& -\left|o_{i}\right|\left[x_{i}(t)-\operatorname{sgn}\left(o_{i}\right) x_{N^{*}}(t)\right],
\end{aligned}
$$

$i \in \mathcal{N}_{n}$, where $a_{i j}$ represents the $(i, j)$ th element in the adjacency matrix $\mathscr{A}$ of $G(\mathscr{A})$ and $o_{i}$ is the $i$ th element in $\alpha$. Combining system (4) with protocol (9) yields

$$
\begin{aligned}
& \dot{X}(t)=U(t) \\
& U(t)=-L_{f f}^{\mathscr{A}} X(t)+\alpha x_{N^{*}}(t),
\end{aligned}
$$

Gauge transformation is a coordinate transformation performed in $\mathbb{R}^{n+1}$, which is represented by matrix $D=$ $\operatorname{diag}\left\{\sigma_{1}, \cdots, \sigma_{n+1}\right\}$. The set consisting of all gauge transformations in $\mathbb{R}^{n+1}$ is represented by $\mathscr{D}=\left\{D=\operatorname{diag}\left\{\sigma_{1}, \cdots\right.\right.$, $\left.\left.\sigma_{n+1}\right\}, \sigma_{i} \in \pm 1, i=1, \cdots, n+1\right\}$. We assume that $G(\mathscr{A})$ is structurally balanced, which means that all nodes can be divided into two parts, $\mathscr{V}_{1}$ and $\mathscr{V}_{2}$, with $\mathscr{V}_{1} \cup \mathscr{V}_{2}=$ $\mathscr{V}, \mathscr{V}_{1} \cap \mathscr{V}_{2}=\phi$ and $a_{i j} \geq 0$ if $\forall i, j \in V_{q}(q \in\{1,2\})$; $a_{i j} \leq 0$ if $\forall i \in V_{q}, j \in V_{r}, q \neq r(q, r \in\{1,2\})$ [34]. It is worth mentioning that if all the semicycles of $G(\mathscr{A})$ are positive, $G(\mathscr{A})$ is structurally balanced [40]. Let $\sigma_{i}=$ 1 and $\sigma_{j}=-1$ correspond to $i \in \mathscr{V}_{1}$ and $j \in \mathscr{V}_{2}$, respectively. For a structural balance graph $G(\mathscr{A})$, the gauge transformation $D$ can transform the adjacency matrix $\mathscr{A}$ of cooperative-competition MASs into a nonnegative matrix $D \mathscr{A D}$. Consequently, the bipartite consensus problem of cooperative-competition systems is transformed into a standard consensus problem of cooperative systems. At the same time, the eigenvalues of $D L D$ under this transformation coincide with those of the laplacian $L$ of the original system. For the transformation

$$
D=\left[\begin{array}{cc}
D_{n} & \mathbf{0}_{n} \\
\mathbf{0}_{n}^{T} & \sigma_{n+1}
\end{array}\right],
$$

where $D_{n}=\operatorname{diag}\left\{\sigma_{1}, \cdots, \sigma_{n}\right\}$, we see that $D^{-1}=D$. For system (4) the following gauge transformation is considered

$$
\pi(t)=D_{n} X(t), \quad D \in \mathscr{D},
$$

and thus, $\dot{\pi}(t)=D_{n} \dot{X}(t)$. One has

$$
\begin{aligned}
\dot{\pi}(t) & =U_{\pi}(t) \\
U_{\pi}(t) & =-D_{n} L_{f f}^{A} D_{n} \pi(t)+D_{n} \alpha \sigma_{n+1} \pi_{N^{*}}(t) .
\end{aligned}
$$

Set the following cost function

$$
\begin{aligned}
& J\left(U_{\pi}(.), \pi(0)\right) \\
& \quad=\int_{0}^{\infty}\left\{\sum_{i=1}^{n}\left[q_{i}\left(\pi_{i}(t)-\pi_{N^{*}}(t)\right)^{2}+r_{i} u_{i}^{2}(t)\right]\right\} d t
\end{aligned}
$$

where $q_{i}>0, r_{i}>0$, which represent the weight of error and the weight of the control cost of follower $i$, respectively. So, the leader-follower multiagent system optimal control problem is suitable for any initial state $X(0) \in \mathbb{R}^{n}$. The goal is to find the minimum optimal control $U^{*}(t)$ of $J(U(),. X(0))$. With (13), the optimal control cost is

$$
\min _{U_{\pi}(t)} J\left(U_{\pi}(t), \pi(0)\right) .
$$

Since $U^{*}(t)$ has been found, by (13), the Laplacian matrix structure can be revealed. Finding the optimal control cost is equivalent to finding the Laplace matrix structure corresponding to the system equation, which is then equivalent to finding the optimal topological structure.

Theorem 2. For (15), the optimal topology with respect to the control input (9) is a star structure, in which any followers are connected only to the leader with weight $\alpha_{i}^{*}=$ $\operatorname{sgn}\left(\sigma_{n+1}\right) \sigma_{i} \sqrt{q_{i} / r_{i}}$. The star structure is the optimal topology solution achieving the bipartite consensus for MASs.

Proof. The control error of follower $i$ is denoted by $\varepsilon_{i}(t)=$ $\pi_{i}(t)-\pi_{N^{*}}(t)$. The control error vector is denoted by $\varepsilon(t)=$ $\left(\varepsilon_{1}(t), \cdots, \varepsilon_{n}(t)\right)^{T}$. The error system of (13) is

$$
\begin{aligned}
\dot{\varepsilon}(t) & =U_{\pi}(t) \\
U_{\pi}(t) & =-D_{n} L_{f f}^{\mathscr{A}} D_{n} \varepsilon(t) .
\end{aligned}
$$

Substituting $\varepsilon(t)$ into $J(U(t), X(0))$, the optimal control problem (15) is transformed to the LQR problem

$$
\min _{U(t)} \int_{0}^{\infty}\left[\varepsilon^{T}(t) Q \varepsilon(t)+U_{\pi}^{T}(t) R U_{\pi}(t)\right] d t
$$


The above formula is derived from (16), where $Q=\operatorname{diag}\left\{q_{1}\right.$, $\left.\cdots, q_{n}\right\}, R=\operatorname{diag}\left\{r_{1}, \cdots, r_{n}\right\}$. Since $\left[I_{n}, 0, \cdots, 0\right]$ is full row rank, system (16) is controllable.

Lemma 1 implies that there exists a positive definite matrix $P \in \mathbb{R}^{n \times n}$, satisfying ARE

$$
P R^{-1} P=Q \text {. }
$$

The optimal feedback gain matrix $D_{n} L_{f f}^{\mathscr{A}^{*}} D_{n}=R^{-1} P$ stabilizes system (16). Premultiplying $R^{-1}$ on both sides of (18) yields

$$
D_{n} L_{f f}^{\mathscr{A}^{*}} D_{n}=\left(R^{-1} Q\right)^{-1 / 2}=\operatorname{diag}\left\{\sqrt{\frac{q_{1}}{r_{1}}}, \cdots, \sqrt{\frac{q_{n}}{r_{n}}}\right\} .
$$

Then $L_{f f}^{\mathscr{A}^{*}}=\operatorname{diag}\left\{\sqrt{q_{1} / r_{1}}, \cdots, \sqrt{q_{n} / r_{n}}\right\}$. Obviously,

$$
L^{\mathscr{A}^{*}}=\left(\begin{array}{cc}
D_{n} L_{f f}^{\mathscr{A}^{*}} D_{n} & -D_{n} \alpha \sigma_{n+1} \\
\mathbf{0}_{N}^{T} & \mathbf{0}
\end{array}\right) .
$$

For all $i \in \mathcal{N}_{n}$, their neighbor nodes constitute only $x_{N^{*}}$. That is, there is no exchange of information among followers. So only followers and leaders have information exchange: $\alpha_{i}^{*}=$ $\sigma_{i} \sqrt{q_{i} / r_{i}} \sigma_{n+1}$.

$G\left(A^{*}\right)$ consisting of $N$ edges satisfies the above condition. Because there is no information exchange among followers, and there is only information exchange between leader and followers; we see that the optimal topology is a star structure. The condition of spanning tree guarantees that the topology structure of the first-order system (10) can achieve the bipartite consensus. Thus star topology is the optimal structure for system to achieve the bipartite consensus.

Remark 3. The optimal topology of the leader-follower MAS is a star structure. The weight of information exchange is $\alpha_{i}^{*}=\sigma_{i} \sqrt{q_{i} / r_{i}} \sigma_{n+1}$. The above arguments show that any element of $L_{f f}^{\mathscr{A}^{*}}$ is nonnegative. The diagonal elements of the coordinate transformation matrix $D_{n}$ depend on $\sigma_{i}= \pm 1$. $\sigma_{n+1}$ represents $\operatorname{sgn}\left(\sigma_{n+1}\right) \cdot 1 . \alpha^{*}$ represents that the exchange of information among topologies is a mixture of weights; i.e., there exists cooperation situation represented by positive weights, and there is competition situation represented by the negative weights. The bipartite consensus means that the individuals achieve the same modulus with opposite sign. For a first-order multiagent system, the structural equilibrium is a necessary and sufficient condition for achieving the bipartite consensus in an undirected connected graph or a directed graph with spanning trees $[35,36]$.

As shown in Figure 1, the optimal structure for the leader (yellow ball) communicating with followers (red ball) is a star topology, and the weights corresponding to each edge are $a_{i}=\operatorname{sgn}\left(\sigma_{n+1}\right) \sigma_{i} \sqrt{q_{i} / r_{i}}$.

3.2. Multileader. The $m$ leaders are denoted by $n+1, \ldots, n+m$. The Laplacian matrix of $G(\mathscr{A})$ is

$$
L^{\mathscr{A}}=\left(\begin{array}{cc}
L_{f f}^{\mathscr{A}} & -L_{f r}^{\mathscr{A}} \\
\mathbf{0}_{m \times n} & \mathbf{0}_{m \times m}
\end{array}\right)
$$

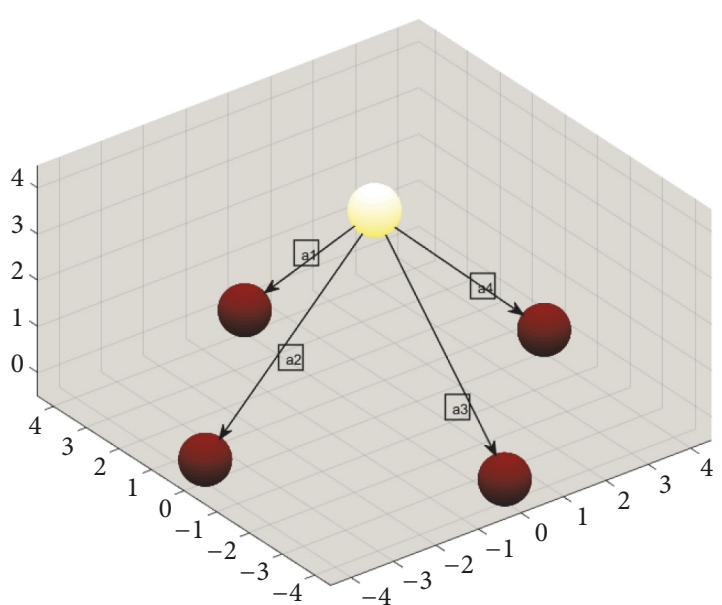

Figure 1

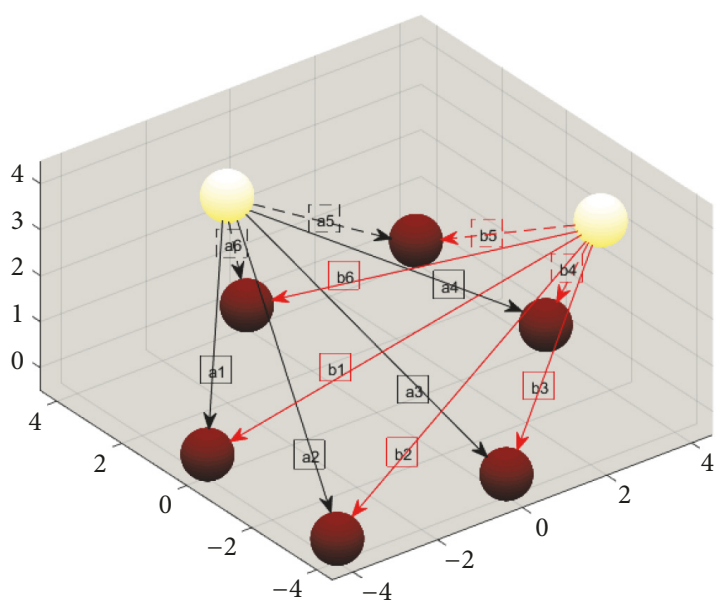

Figure 2

where $L_{f f}^{\mathscr{A}} \in \mathbb{R}^{n \times n}, L_{f r}^{\mathscr{A}} \in \mathbb{R}^{n \times m} . X_{f}(t)=\left[x_{1}(t), \cdots, x_{n}(t)\right]^{T}$, $X_{r}(t)=\left[x_{n+1}(t), \cdots, x_{n+m}(t)\right]^{T}$. It follows that

$$
\begin{aligned}
& \dot{X}_{f}(t)=-L_{f f}^{\mathscr{A}} X_{f}(t)+L_{f r}^{\mathscr{A}} X_{r}(t) \\
& \dot{X}_{r}(t)=\mathbf{0}_{m} .
\end{aligned}
$$

Then the gauge transformation yields

$$
\dot{\pi}_{f}(t)=-D_{n} L_{f f}^{\mathscr{A}} D_{n} \pi_{f}(t)+D_{n} L_{f r}^{\mathscr{A}} D_{m} \pi_{r}(t),
$$

where $D_{m}=\operatorname{diag}\left(\sigma_{n+1}, \cdots, \sigma_{n+m}\right)$.

Theorem 4. With respect to the control input (9), the optimal topology structure of leader-follower MASs $(m>1)$ is a star composite topology, which is the best form of information exchange between followers and leaders. The weight between leaders and followers is the solution of convex hull: $\min _{b_{i}}\left\{\pi_{i}(0)-\sqrt{r_{i} / q_{i}} b_{i}^{*} \pi_{r}(0)\right\}^{2} ; b_{i}^{*} \mathbf{1}_{m}=\sqrt{q_{i} / r i}$, which is a star composite structure and is the optimal structure solution achieving the bipartite consensus of MASs, as shown in Figure 2. 
Proof. From [41], if $D_{n} L_{f f}^{\mathscr{A}} D_{n}$ is an invertible matrix, then $\pi_{f}(t)$ tends to $\pi_{f}^{c}=\left(D_{n} L_{f f}^{\mathscr{A}} D_{n}\right)^{-1} D_{n} L_{f r}^{\mathscr{A}} D_{m} \pi_{r}(0)$. Therefore, the cost function is

$$
\begin{aligned}
& J\left(U_{\pi}(t), \pi(0)\right) \\
& \quad=\int_{0}^{\infty}\left[\left(\pi_{f}(t)-\pi_{f}^{c}\right)^{T} Q\left(\pi_{f}(t)-\pi_{f}^{c}\right)\right. \\
& \left.\quad+U_{\pi}(t)^{T} R U_{\pi}(t)\right] d t,
\end{aligned}
$$

where $Q$ and $R$ are both $N$-dimensional positive definite diagonal matrices; $Q=\operatorname{diag}\left\{q_{1}, q_{2}, \cdots, q_{n}\right\}, R=$ $\operatorname{diag}\left\{r_{1}, r_{2}, \cdots, r_{n}\right\}$. Similar to the proof of Theorem 2 , the optimal control problem reads

$$
J\left(U^{*}(.), \pi(0)\right)=\min _{U_{\pi}(.)} J\left(U_{\pi}(.), \pi(0)\right) .
$$

It follows from (22) and Lemma 1 that

$$
\begin{aligned}
L_{f f}^{\mathscr{A}^{*}} & =\operatorname{diag}\left\{\sqrt{\frac{q_{1}}{r_{1}}}, \ldots, \sqrt{\frac{q_{n}}{r_{n}}}\right\}, \\
D_{n} L_{f r}^{\mathscr{A}^{*}} D_{m} & =\left(\begin{array}{c}
b_{1}^{*} \\
\ldots \\
b_{n}^{*}
\end{array}\right),
\end{aligned}
$$

where $\mathbf{b}_{i}^{*} \in \mathbb{R}^{1 \times m}\left(i \in \mathcal{N}_{n}\right)$ is the solution of the convex hull.

$$
\begin{gathered}
\min _{b_{i}}\left\{\pi_{i}(0)-\sqrt{\frac{r_{i}}{q_{i}}} b_{i}^{*} \pi_{r}(0)\right\}^{2} \\
b_{i}^{*} \mathbf{1}_{m}=\sqrt{\frac{q_{i}}{r i}} .
\end{gathered}
$$

From (27), we see that followers only communicate with each pilot, and accordingly the corresponding optimal topology is a composite structure based on a star topology. Thus the composite topology is the optimal structure achieving bipartite consensus for the first-order leader-follower multiagent system $(m>1)$.

The picture in Figure 2 depicts a star-shaped composite structure consisting of two pilots (yellow ball) and six followers (red ball). There is no communication between these two leaders, and each follower only receives neighbor information from leaders. The weight satisfies $a_{i}+b_{i}=\sqrt{q_{i} / r_{i}}$ and $\min _{b_{i}}\left\{\pi_{i}(0)-\sqrt{r_{i} / q_{i}}\left(a_{i}+b_{i}\right) \pi_{r}(0)\right\}^{2}$.

\section{LQR Based Optimal Topology of Second- Order Hybrid-Weighted Multiagent Systems}

4.1. Single Leader. The second-order leader-follower multiagent system is

$$
\begin{aligned}
& x_{i}(t)=v_{i}(t), \\
& \dot{v}_{i}(t)=u_{i}(t),
\end{aligned}
$$$$
i \in \mathcal{N}_{n}
$$

$$
\begin{aligned}
& \dot{x}_{N^{*}}(t)=v_{N^{*}}(t), \\
& \dot{v}_{N^{*}}(t)=0,
\end{aligned}
$$

where $x_{i}(t) \in \mathbb{R}, v_{i}(t) \in \mathbb{R}, u_{i}(t) \in \mathbb{R}$ denote the state, velocity, and control of follower $i$, respectively. $x_{N^{*}}(t) \epsilon$ $\mathbb{R}, v_{N^{*}}(t) \in \mathbb{R}$ represent the leader's state and velocity information, respectively. In what follows, we denote $X(t)=$ $\left(x_{1}(t), \cdots, x_{n}(t)\right)^{T}, V(t)=\left(v_{1}(t), \cdots, v_{n}(t)\right)^{T}, U(t)=\left(u_{1}(t)\right.$, $\left.\cdots, u_{n}(t)\right)^{T}$. The second-order MASs consensus is asymptotically achieved if

$$
\begin{aligned}
& \lim _{\mathrm{t} \longrightarrow \infty}\left|x_{i}(t)-x_{N^{*}}(t)\right|=0 \\
& \lim _{\mathrm{t} \longrightarrow \infty}\left|v_{i}(t)-v_{N^{*}}(t)\right|=0, \quad i \in \mathcal{N}_{n}
\end{aligned}
$$

In this section, let us consider the following agreement

$$
\begin{aligned}
u_{i}= & -\sum_{j=1, j \neq i}^{n}\left|a_{i j}\right|\left[x_{i}-\operatorname{sgn}\left(a_{i j}\right) x_{j}\right] \\
& -\left|b_{i}\right|\left[x_{i}-\operatorname{sgn}\left(b_{i}\right) x_{N^{*}}\right] \\
& -\sum_{j=1, j \neq i}^{n}\left|w_{i j}\right|\left[v_{i}-\operatorname{sgn}\left(w_{i j}\right) v_{j}\right] \\
& -\left|d_{i}\right|\left[v_{i}-\operatorname{sgn}\left(d_{i}\right) v_{N^{*}}\right],
\end{aligned}
$$

where $a_{i j}, w_{i j}$ represent the $(i, j)$ element of adjacency matrix $\mathscr{A}$ and $\mathscr{W}$ of the directed graph $G(A)$ and $G(W)$, respectively, and $b_{i}, d_{i}$ are, respectively, the ith elements of $\mathbf{b}, \mathbf{d}$. With protocol (30), system (28) is written in a matrix form

$$
\begin{aligned}
\left(\begin{array}{c}
\dot{X}(t) \\
\dot{V}(t)
\end{array}\right)= & G\left(\begin{array}{c}
X(t) \\
V(t)
\end{array}\right)+H U(t) \\
U(t)= & -L_{f f}^{A} X(t)-L_{f f}^{W} V(t)+\mathbf{b} x_{N^{*}}(t) \\
& +\mathbf{d} v_{N^{*}}(t),
\end{aligned}
$$

where

$$
\begin{aligned}
& G=\left(\begin{array}{cc}
0_{n \times n} & I_{n} \\
0_{n \times n} & 0_{n \times n}
\end{array}\right), \\
& H=\left(\begin{array}{c}
0_{n \times n} \\
I_{n}
\end{array}\right) .
\end{aligned}
$$

By (11), the gauge transformation of (28) is

$$
\begin{aligned}
& \pi(t)=D_{n} X(t), \\
& \beta(t)=D_{n} V(t),
\end{aligned}
$$

$D \in \mathscr{D}$.

From $D=D^{-1}, X(t)=D_{n} \pi(t), V(t)=D_{n} \beta(t)$, one has

$$
\dot{\pi}(t)=D_{n} \dot{X}(t)=D_{n} V(t)=\beta(t) .
$$


It follows that

$$
\begin{aligned}
& \dot{\pi}_{i}(t)=\beta_{i}(t), \\
& \dot{\beta}_{i}(t)=\gamma_{i}(t),
\end{aligned}
$$

$$
i \in \mathcal{N}_{N}
$$

$$
\begin{aligned}
& \dot{\pi}_{N^{*}}(t)=\beta_{N^{*}}(t), \\
& \gamma_{N^{*}}(t)=0 .
\end{aligned}
$$

For (31), $U(t)=-L_{f f}^{\mathscr{A}} X(t)-L_{f f}^{\mathscr{W}} V(t)+\mathbf{b} x_{N^{*}}(t)+\mathbf{d} v_{N^{*}}(t)$. Therefore

$$
\begin{aligned}
\gamma(t)= & D_{n} \dot{V}(t) \\
= & -D_{n} L_{f f}^{\mathscr{A}} D_{n} \pi(t)-D_{n} L_{f f}^{\mathscr{W}} D_{n} \beta(t) \\
& +D_{n} \mathbf{b} \sigma_{n+1} \pi_{N^{*}}(t)+D_{n} \mathbf{d} \sigma_{n+1} \beta_{N^{*}}(t),
\end{aligned}
$$

where $D_{n}=\operatorname{diag}\left\{\sigma_{1}, \cdots, \sigma_{n}\right\}$. We get

$$
\begin{aligned}
\left(\begin{array}{c}
\dot{\pi}(t) \\
\dot{\beta}(t)
\end{array}\right)= & \left(I_{2} \otimes D_{n}\right) G\left(I_{2} \otimes D_{n}\right)\left(\begin{array}{c}
\pi(t) \\
\beta(t)
\end{array}\right) \\
& +\left(I_{2} \otimes D_{n}\right) H D_{n} \gamma(t) \\
\gamma(t)= & -D_{n} L_{f f}^{\mathscr{A}} D_{n} \pi(t)-D_{n} L_{f f}^{\mathscr{W}} D_{n} \beta(t) \\
& +D_{n} \mathbf{b} \sigma_{n+1} \pi_{N^{*}}(t)+D_{n} \mathbf{d} \sigma_{n+1} \beta_{N^{*}}(t) .
\end{aligned}
$$

By (37), the optimal control problem of (35) is

$$
\begin{aligned}
& \min _{\gamma(t)} J\left(\gamma(t),[\pi(0), \beta(0)]^{T}\right), \\
& J\left(\gamma(t),[\pi(0), \beta(0)]^{T}\right)=\int_{0}^{\infty} \sum_{i=1}^{N} r_{i} \gamma_{i}^{2}(t) d t \\
& \quad+\int_{0}^{\infty} \sum_{i=1}^{n} q_{i}\left(\pi_{i}(t)-\pi_{N^{*}}(t)\right)^{2} d t \\
& \quad+\int_{0}^{\infty} \sum_{i=1}^{n} q_{i}\left(\beta_{i}(t)-\beta_{N^{*}}(t)\right)^{2} d t
\end{aligned}
$$

where $q_{i}>0, r_{i}>0$. Therefore, finding the optimal control $\gamma^{*}(t)$ is equivalent to finding the optimal topology structures which correspond to their velocity and state graphs $\left(G\left(\mathscr{A}^{*}\right), W\left(\mathscr{A}^{*}\right)\right)$, respectively.

Note that the structure is included, respectively, in Laplacian matrices $D_{n} L^{\mathscr{A}^{*}} D_{n}$ and $D_{n} L^{\mathscr{W}^{*}} D_{n}$.

Theorem 5. For the optimal control problem (38) with the control input (30), the optimal topology is an unevenly weighted star structure, in which each follower $i$ is only connected with the leader. The weight of the state graph is $\sigma_{i} \sigma_{n+1} \sqrt{q_{i} / r_{i}}$, and the weight in velocity graph is $\sigma_{i} \sigma_{n+1} \sqrt{2 \sqrt{q_{i} / r_{i}}+q_{i} / r_{i}}$. The star structure is the optimal topology solution achieving the bipartite consensus of MASs.
Proof. The consensus error system of (35) is as follows

$$
\varepsilon(t)=\left(\begin{array}{c}
\pi(t) \\
\beta(t)
\end{array}\right)-\left[\mathbf{1}_{n}^{T} \otimes \pi_{N^{*}}(t), \mathbf{1}_{n}^{T} \otimes \beta_{N^{*}}(t)\right]^{T} .
$$

The corresponding error power system is

$$
\begin{aligned}
\dot{\varepsilon}(t)= & \left(I_{2} \otimes D_{n}\right) G\left(I_{2} \otimes D_{n}\right) \varepsilon(t) \\
& +\left(I_{2} \otimes D_{n}\right) H D_{n} \gamma(t) \\
\gamma(t)= & -K \varepsilon(t),
\end{aligned}
$$

where $K=\left[D_{n} L_{f f}^{\mathscr{A}} D_{n}, D_{n} L_{f f}^{\mathscr{W}} D_{n}\right]$. Therefore, the optimal control problem (38) is transformed into the following LQR problem

$$
\min _{\gamma(t)} \int_{0}^{\infty}\left[\varepsilon^{T}(t)\left(I_{2} \otimes Q\right) \varepsilon(t)+\gamma^{T}(t) R \gamma(t)\right] d t
$$

subject to (41), where $Q=\operatorname{diag}\left\{q_{1}, \cdots, q_{n}\right\}, R=\operatorname{diag}\left\{r_{1}\right.$, $\left.\cdots, r_{n}\right\}$.

$$
\begin{gathered}
\operatorname{rank}\left[\left(I_{2} \otimes D_{n}\right) H D_{n},\left(I_{2} \otimes D_{n}\right) G H D_{n}, \cdots,\left(I_{2} \otimes D_{n}\right)\right. \\
\left.\cdot G^{2 n-1} H D_{n}\right]=\operatorname{rank}\left[H, G H, \cdots, G^{2 n-1} H\right]=2 n .
\end{gathered}
$$

Since the above matrix is full row rank, system (38) is controllable.

Similar to Theorem 2, the ARE equation corresponding to $(42)$ is

$$
\begin{aligned}
& P\left(I_{2} \otimes D_{n}\right) H R^{-1} H^{T}\left(I_{2} \otimes D_{n}\right) P \\
&= P\left(I_{2} \otimes D_{n}\right) G\left(I_{2} \otimes D_{n}\right) \\
& \quad+\left(I_{2} \otimes D_{n}\right) G^{T}\left(I_{2} \otimes D_{n}\right) P+I_{2} \otimes Q .
\end{aligned}
$$

This equation has a unique positive definite solution. In addition, the optimal control is $\gamma^{*}(t)=-R^{-1} D_{n} H^{T}\left(I_{2} \otimes\right.$ $\left.D_{n}\right) P \varepsilon(t)$. In (44), the structure forms of matrices $G, Q, R, H$ imply that $P$ is a symmetric matrix. It can be written as

$$
P=\left(\begin{array}{ll}
P_{11} & P_{12} \\
P_{12}^{T} & P_{22}
\end{array}\right)
$$

where $P_{11} \in \mathbb{R}^{n \times n}, P_{22} \in \mathbb{R}^{n \times n}$ are both symmetric. $P_{12}=$ $P_{12}^{T} \in \mathbb{R}^{n \times n}$. It follows that

$$
\begin{aligned}
K^{*} & =\left[D_{n} L_{f f}^{\mathscr{A}^{*}} D_{n}, D_{n} L_{f f}^{\mathscr{W}^{*}} D_{n}\right] \\
& =R^{-1} D_{n} H^{T}\left(I_{2} \otimes D_{n}\right) P=\left[R^{-1} P_{12}^{T}, R^{-1} P_{22}\right] .
\end{aligned}
$$

From (44), we have

$$
\begin{gathered}
P_{12} R^{-1} P_{22}=P_{11}=P_{22} R^{-1} P_{12}^{T}, \\
P_{12}^{T}+P_{12}+Q=P_{22} R^{-1} P_{22}, \\
Q=P_{12} R^{-1} P_{12}^{T} .
\end{gathered}
$$




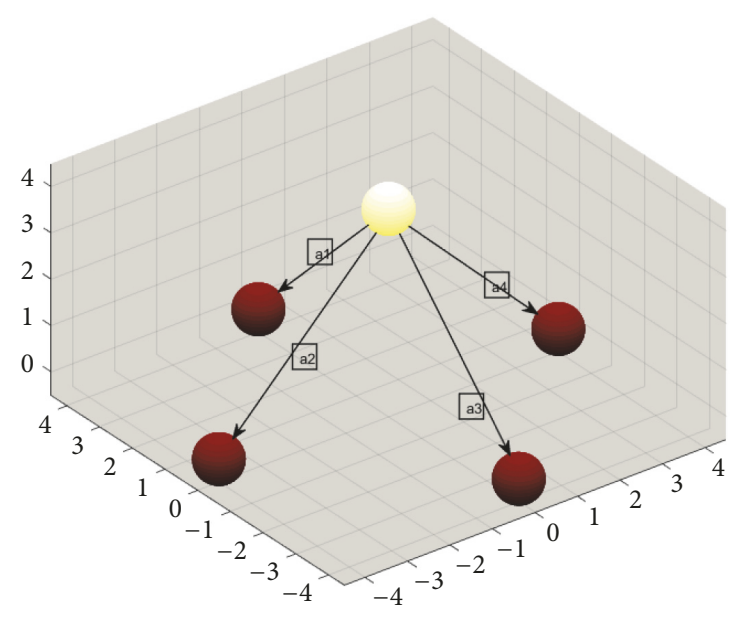

FIgURE 3

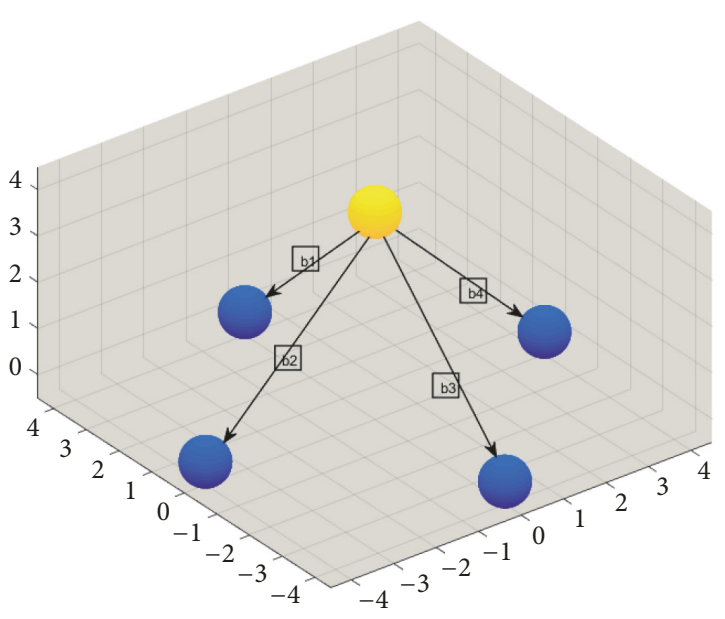

FIGURE 4

Premultiplying $R^{-1}$ on both sides of (48), we have

$$
\begin{aligned}
L_{f f}^{\mathscr{A}} & =D_{n}\left(R^{-1} Q\right)^{1 / 2} D_{n} \\
L_{f f}^{\mathscr{W}^{*}} & =D_{n}\left(R^{-1}\left(2 P_{12}+Q\right)\right)^{1 / 2} D_{n},
\end{aligned}
$$

where $D_{i} b_{i}^{*} \sigma_{n+1}=\sqrt{q_{\mathrm{i}} / r_{i}}, D_{i} d_{i}^{*} \sigma_{n+1}=\sqrt{2 \sqrt{q_{i} / r_{i}}+q_{i} / r_{i}}$. Therefore

$$
\begin{aligned}
L^{\mathscr{A}^{*}} & =\left(\begin{array}{cc}
D_{n} L_{f f}^{\mathscr{A}^{*}} D_{n} & -D_{n} \mathbf{b}^{*} \sigma_{n+1} \\
\mathbf{0}_{n}^{T} & 0
\end{array}\right) \\
L^{\mathscr{W}^{*}} & =\left(\begin{array}{cc}
D_{n} L_{f f}^{\mathscr{W}^{*}} D_{n} & -D_{n} \mathbf{d}^{*} \sigma_{n+1} \\
\mathbf{0}_{n}^{T} & 0
\end{array}\right) .
\end{aligned}
$$

In (50), $\mathbf{b}^{*}=D_{n} L_{f f}^{\mathscr{A}}\left(I_{n} \otimes \sigma_{n+1}\right), \mathbf{d}^{*}=D_{n} L_{f f}^{\mathscr{W}^{*}}\left(I_{n} \otimes \sigma_{n+1}\right)$, which means that the optimal topology is a nonuniformly weighted star topology; i.e., follower $i$ has only information exchange with the single leader; the weights on the state graph and velocity graph are $\sigma_{i} \sigma_{n+1} \sqrt{q_{i} / r_{i}}, \sigma_{i} \sigma_{n+1} \sqrt{2 \sqrt{q_{i} / r_{i}}+q_{i} / r_{i}}$, respectively.

As a consequence, the star topology with uneven hybrid weights can make system (28) achieve both the bipartite consensus and the optimal topology.

As shown in Figures 3 and 4, the unevenly hybridweighted star structure is the optimal communication topology consisting of leader (yellow ball) and followers (red and blue ball). The star topology has the same position and velocity topology structure with different weights. The corresponding weight in the position and velocity topology is $\operatorname{sgn}\left(\sigma_{n+1}\right) \sigma_{i} \sqrt{q_{i} / r_{i}}$ and $\operatorname{sgn}\left(\sigma_{n+1}\right) \sigma_{i} \sqrt{2 \sqrt{q_{i} / r_{i}}+q_{i} / r_{i}}$, respectively.

4.2. Multileader. Consider a leader-follower MAS with multiple leaders $(m>1)$.

$$
\begin{aligned}
& \dot{x}_{i}(t)=v_{i}(t), \\
& \dot{v}_{i}(t)=u_{i}(t), \\
& \qquad i \in \mathscr{I}_{n}, \\
& \dot{x}_{r}(t)=v_{r}(t), \\
& \dot{v}_{r}(t)=0,
\end{aligned}
$$

where $x_{i}(t) \in \mathbb{R}, v_{i}(t) \in \mathbb{R}, u_{i}(t) \in \mathbb{R}$ denote the state, velocity, and control of follower $i$, respectively. $x_{r}(t) \in$ $\mathbb{R}, v_{r}(t) \in \mathbb{R}$ represent the leaders' state and velocity information, respectively. In what follows, we denote $X_{f}(t)=$ $\left[x_{1}(t), \cdots, x_{n}(t)\right]^{T}, X_{r}(t)=\left[x_{n+1}(t), \cdots, x_{n+m}(t)\right]^{T}, V_{f}(t)=$ $\left[v_{1}(t), \cdots, v_{n}(t)\right]^{T}, V_{r}(t)=\left[v_{n+1}(t), \cdots, v_{n+m}(t)\right]^{T}, U(t)=$ $\left[u_{1}(t), \cdots, u_{n}(t)\right]^{T}$.

The $m$ leaders are indicated by $n+1, \ldots, n+m$. The Laplacian matrices of $G(\mathscr{A})$ and $G(\mathscr{W})$ are

$$
\begin{aligned}
L^{\mathscr{A}} & =\left(\begin{array}{cc}
L_{f f}^{\mathscr{A}} & -L_{f r}^{\mathscr{A}} \\
\mathbf{0}_{m \times n} & \mathbf{0}_{m \times m}
\end{array}\right), \\
L^{\mathscr{W}} & =\left(\begin{array}{cc}
L_{f f}^{\mathscr{V}} & -L_{f r}^{\mathscr{W}} \\
\mathbf{0}_{m \times n} & \mathbf{0}_{m \times m}
\end{array}\right),
\end{aligned}
$$

where $L_{f f}^{\mathscr{A}} \in \mathbb{R}^{n \times n}, L_{f r}^{\mathscr{A}} \in \mathbb{R}^{n \times m}, L_{f f}^{\mathscr{W}} \in \mathbb{R}^{n \times n}$, and $L_{f r}^{\mathscr{W}} \in \mathbb{R}^{n \times m}$. Consider the following linear protocol

$$
u_{i}(t)=-\sum_{j=1, j \neq i}^{n}\left\{\left|a_{i j}\right|\left[x_{i}(t)-\operatorname{sgn}\left(\mathrm{a}_{\mathrm{ij}}\right) x_{j}(t)\right]+\left|w_{i j}\right|\left[v_{i}(t)-\operatorname{sgn}\left(\mathrm{w}_{\mathrm{ij}}\right) v_{j}(t)\right]\right\}
$$




$$
-\sum_{r=n+1}^{n+m}\left\{\left|b_{i r}\right|\left[x_{i}(t)-\operatorname{sgn}\left(\mathrm{b}_{\mathrm{ir}}\right) x_{r}(t)\right]+\left|d_{i r}\right|\left[v_{i}(t)-\operatorname{sgn}\left(\mathrm{d}_{\mathrm{ir}}\right) v_{r}(t)\right]\right\}
$$

where $a_{i j}, w_{i j}$ denote the $(i, j)$ th element of $\mathscr{A}$ and $\mathscr{W}$, respectively. Then $b_{i r}, d_{i r}$ are the $(i, r)$ th element of $L_{f r}^{\mathscr{A}}$ and $L_{f r}^{\mathscr{W}}$, respectively. In view of system (51) and protocol (53), we have

$$
\begin{aligned}
\left(\begin{array}{c}
\dot{X}_{f}(t) \\
\dot{V}_{f}(t)
\end{array}\right)= & G\left(\begin{array}{c}
X_{f}(t) \\
V_{f}(t)
\end{array}\right)+H U(t) \\
U(t)= & -L_{f f}^{\mathscr{A}} X_{f}(t)-L_{f f}^{\mathscr{W}} V_{f}(t)+L_{f r}^{\mathscr{A}} X_{r}(t) \\
& +L_{f r}^{\mathscr{W}} X_{r}(t),
\end{aligned}
$$

where

$$
\begin{aligned}
& G=\left(\begin{array}{cc}
0_{n \times n} & I_{n} \\
0_{n \times n} & 0_{n \times n}
\end{array}\right), \\
& H=\left(\begin{array}{c}
0_{n \times n} \\
I_{n}
\end{array}\right) .
\end{aligned}
$$

Similar to the gauge transformations implemented in Theorem 4, we see that

$$
\begin{aligned}
\gamma(t) & =D_{n} \dot{v}(t) \\
& =-D_{n} L_{f f}^{\mathscr{A}} D_{n} \pi_{f}(t)-D_{n} L_{f f}^{\mathscr{V}} D_{n} \beta_{f}(t)
\end{aligned}
$$

$$
+D_{n} L_{f r}^{A} D_{m} \pi_{r}(t)+D_{n} L_{f r}^{W} D_{m} \beta_{r}(t)
$$

where $D_{n}=\operatorname{diag}\left\{\sigma_{1}, \cdots, \sigma_{n}\right\}, D_{m}=\operatorname{diag}\left\{\sigma_{n+1}, \cdots, \sigma_{n+m}\right\}$. Hence

$$
\begin{aligned}
\left(\begin{array}{c}
\dot{\pi}_{f}(t) \\
\dot{\beta}_{f}(t)
\end{array}\right)= & \left(I_{2} \otimes D_{n}\right) G\left(I_{2} \otimes D_{n}\right)\left(\begin{array}{c}
\pi_{f}(t) \\
\beta_{f}(t)
\end{array}\right) \\
& +\left(I_{2} \otimes D_{n}\right) H D_{n} \gamma(t), \\
\gamma(t)= & D_{n} \dot{v}(t) \\
= & -D_{n} L_{f f}^{\mathscr{A}} D_{n} \pi_{f}(t)-D_{n} L_{f f}^{\mathscr{W}} D_{n} \beta_{f}(t) \\
& +D_{n} L_{f r}^{\mathscr{A}} D_{m} \pi_{r}(t)+D_{n} L_{f r}^{\mathscr{W}} D_{m} \beta_{r}(t) .
\end{aligned}
$$

The optimal control problem is considered subject to (57)

$$
\min _{\gamma(t)} J\left(\gamma(t),\left[\pi^{T}(0), \beta^{T}(0)\right]^{T}\right) .
$$

In a second-order leader-follower MAS with $m>1$ leaders, we assume that the position state tends to $\pi_{\xi}(t)$ and the velocity state tends to $\beta_{\xi}(t)$. Define the cost function

$$
J(\gamma(t), \pi(0))=\int_{0}^{\infty}\left\{\sum_{i=1}^{n}\left[q_{i}\left(\left(\pi_{i}(t)-\pi_{\xi}(t)\right)^{2}+\left(\beta_{i}(t)-\beta_{\xi}(t)\right)^{2}\right)+r_{i} \gamma_{i}^{2}(t)\right]\right\} d t
$$

where $q_{i}>0, r_{i}>0$.

Theorem 6. For the optimal control problem (58) with the control input (30), the optimal structure of leader-follower MASs $(m>1)$ is an unevenly hybrid-weighted star composite topology consisting of a star structure formed by followers and leaders. In addition, the structure is the optimal topology solution achieving the bipartite consensus of MASs, as shown in Figure 5.

Proof. For system (54), the consensus error vector is defined by

$$
\varepsilon(t)=\left(\begin{array}{c}
\pi_{f}(t)-\pi_{\xi}(t) \\
\beta_{f}(t)-\beta_{\xi}(t)
\end{array}\right) .
$$

Similar to Theorem 4, the corresponding error dynamics system is

$$
\dot{\varepsilon}(t)=\left(I_{2} \otimes D_{n}\right) G\left(I_{2} \otimes D_{n}\right) \varepsilon(t)
$$

$$
\begin{aligned}
& +\left(I_{2} \otimes D_{n}\right) H D_{n} \gamma(t), \\
\gamma(t)= & -K \varepsilon(t),
\end{aligned}
$$

where $K=\left[D_{n} L_{f f}^{\mathscr{A}} D_{n}, D_{n} L_{f f}^{\mathscr{W}} D_{n}\right]$. Therefore, the optimal control problem (53) is converted to the following LQR problem

$$
\min _{\gamma(t)} \int_{0}^{\infty}\left[\varepsilon^{T}(t)\left(I_{2} \otimes Q\right) \varepsilon(t)+\gamma^{T}(t) R \gamma(t)\right] d t
$$

subject to $(58)$,

where $Q=\operatorname{diag}\left\{q_{1}, \cdots, q_{n}\right\}, R=\operatorname{diag}\left\{r_{1}, \cdots, r_{n}\right\}$. Similar to Theorem 5, we have

$$
D_{n}(i) b_{i}^{*} D_{m}=\sqrt{\frac{q_{i}}{r_{i}}}
$$




$$
D_{n}(i) d_{i}^{*} D_{m}=\sqrt{2 \sqrt{\frac{q_{i}}{r_{i}}+\frac{q_{i}}{r_{i}}}} .
$$

We have the following matrices:

$$
\begin{aligned}
L^{A^{*}} & =\left(\begin{array}{cc}
D_{n} L_{f f}^{A^{*}} D_{n} & -D_{n} L_{f r}^{A^{*}} D_{m} \\
\mathbf{0}_{n}^{T} & 0
\end{array}\right), \\
L^{W^{*}} & =\left(\begin{array}{cc}
D_{n} L_{f f}^{W^{*}} D_{n} & -D_{n} L_{f r}^{\mathscr{W}^{*}} D_{m} \\
\mathbf{0}_{n}^{T} & 0
\end{array}\right) .
\end{aligned}
$$

From $\mathbf{b}^{*}=D_{n} L_{f f}^{\mathscr{A}^{*}} D_{m}, \mathbf{d}^{*}=D_{n} L_{f f}^{\mathscr{W}^{*}} D_{m}$, we see that the optimal topology of the leaders-followers MASs $(m>1)$ is the unevenly hybrid-weighted star topology, in which follower $i$ only exchanges information with the leaders. Then, the unevenly hybrid-weighted star composite structure is the optimal topology for system (51) to achieve the bipartite consensus.

Remark 7. For a leader-follower MAS $(m>1)$, the leaders' states drive the followers to make their states tend to a fixed value. In the above arguments, $\pi_{\xi}(t)$ and $\beta_{\xi}(t)$ described the final converge value of followers' position and velocity, respectively [41].

As shown in Figures 5 and 6, for a second-order leadersfollowers multiagent system $(m>1)$, the optimal communication topology is a hybrid-weighted weight star composite structure. In this structure, there is no information exchange between leaders; and the connection weights between leaders and followers in position graph are different from those in velocity graph.

\section{LQR Based Optimal Topology of General Linear Hybrid-Weighted Leader-Follower Multiagent Systems}

The general linear leader-follower multiagent system is defined as follows

$$
\begin{aligned}
\dot{x}_{i}(t) & =A x_{i}(t)+B u_{i}(t), \quad i \in \mathcal{N}_{n} \\
\dot{x}_{N^{*}}(t) & =A x_{N^{*}}(t),
\end{aligned}
$$

where $x_{i}(t) \in \mathbb{R}$ and $u_{i}(t) \in \mathbb{R}$ denote the state and control of follower $i\left(i \in \mathcal{N}_{n}\right)$, respectively. $x_{N^{*}}(t)$ represents the leader's position. $X(t)=\left(x_{1}(t), \cdots, x_{n}(t)\right)^{T}, U(t)=$ $\left(u_{1}(t), \cdots, u_{n}(t)\right)^{T}$. The Laplace matrix $L^{\mathscr{A}^{n}}$ is defined as Theorem 2. Consider the common linear protocol

$$
\begin{aligned}
u_{i}(t)= & -\sum_{j=1}^{n}\left|a_{i j}\right|\left[x_{i}(t)-\operatorname{sgn}\left(a_{i j}\right) x_{j}(t)\right] \\
& -\left|o_{i}\right|\left[x_{i}(t)-\operatorname{sgn}\left(o_{\mathrm{i}}\right) x_{N^{*}}(t)\right], \quad i \in N_{n},
\end{aligned}
$$

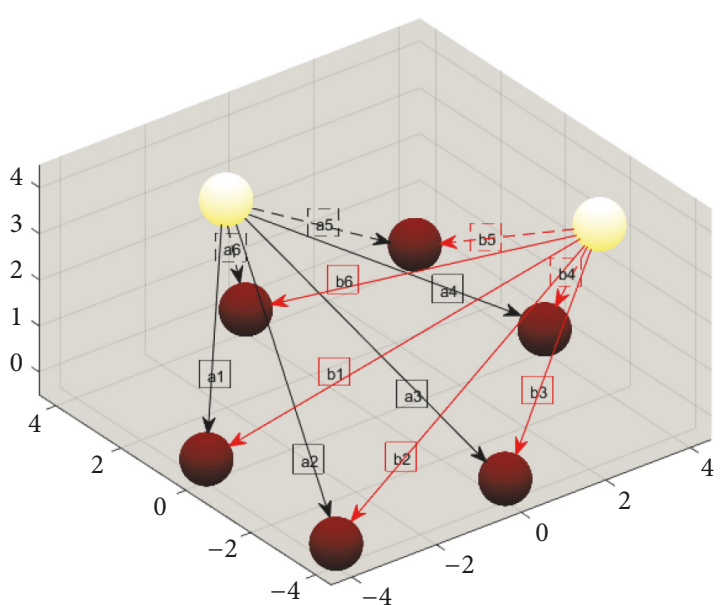

Figure 5

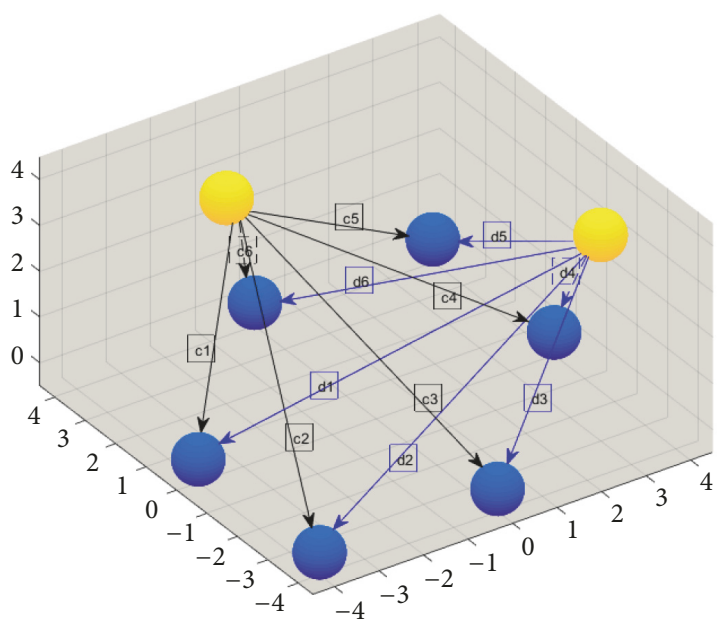

Figure 6

where $a_{i j}$ represents the $(i, j)$ th element of adjacency matrix $\mathscr{A}$ of $G(\mathscr{A}) ; o_{i}$ is the $i$ th element of $\alpha$. By (65) and (66)

$$
\begin{aligned}
& \dot{X}(t)=I_{n} \otimes A X(t)+I_{n} \otimes B U(t) \\
& U(t)=-L_{f f}^{\mathscr{A}} X(t)+\alpha x_{N^{*}}(t) .
\end{aligned}
$$

As in Theorem 2, D is a diagonal matrix with diagonal elements \pm 1 .

$$
D^{*}=\left(\begin{array}{cc}
D_{n}^{*} & \mathbf{0}^{T} \\
\mathbf{0} & D_{n+1}
\end{array}\right)
$$

where $D_{n}^{*}=\operatorname{diag}\left\{D_{1}, \cdots, D_{n}\right\}$. For (67), consider the gauge transformation

$$
\pi(t)=D_{n}^{*} X(t), \quad D_{n}^{*} \in D^{*}
$$

From $D^{-1}=D$, we have

$$
\dot{\pi}(t)=D_{n}^{*} \dot{X}(t),
$$


and then

$$
\begin{aligned}
\dot{X}(t)= & I_{n} \otimes A X(t)-I_{n} \otimes B L_{f f}^{\mathscr{A}} X(t)+I_{n} \\
& \otimes B \alpha x_{N^{*}}(t) .
\end{aligned}
$$

Therefore

$$
\begin{aligned}
\dot{\pi}(t)= & D_{n}^{*} \dot{X}(t) \\
= & D_{n}^{*} I_{n} \otimes A D_{n}^{*} \pi(t)-D_{n}^{*} I_{n} \otimes B L_{f f}^{\mathscr{A}} D_{n}^{*} \pi(t) \\
& +D_{n}^{*} I_{n} \otimes B \alpha D_{n+1} \pi_{N^{*}}(t) .
\end{aligned}
$$

Assuming that $(A, B)$ is controllable, we consider the following cost function

$$
\begin{aligned}
J( & \left.U_{\pi}(.), \pi(0)\right) \\
& =\int_{0}^{\infty}\left\{\sum_{i=1}^{n}\left[q_{i}\left(\pi_{i}(t)-\pi_{N^{*}}(t)\right)^{2}+r_{i} u_{i}^{2}(t)\right]\right\} d t,
\end{aligned}
$$

where $q_{i}>0, r_{i}>0$ represent the weight of error and control cost of follower $i$, respectively. Find the optimal control cost under (72)

$$
\min _{U .(t)} J\left(U_{\pi}(t), \pi(0)\right) .
$$

Theorem 8. For system (65) with the control input (66), the star structure is the optimal topology solution to achieve the bipartite consensus. For this star structure, any follower $i(i \in$ $\mathcal{N}_{n}$ ) is only connected to the leader.

Proof. We denote the control error of follower $i$ with $\varepsilon_{i}(t)=$ $\pi_{i}(t)-\pi_{N^{*}}(t)$. Let $\varepsilon(t)=\left(\varepsilon_{1}(t), \cdots, \varepsilon_{N}(t)\right)^{T}$ represent the control error vector. Then the error system of (72) is

$$
\begin{aligned}
\dot{\varepsilon}(t) & =D_{n}^{*} I_{n} \otimes A D_{n}^{*} \varepsilon(t)+D_{n}^{*} I_{n} \otimes B D_{n}^{*} U_{\pi}(t) \\
U_{\pi}(t) & =-D_{n}^{*} L_{f f}^{\mathscr{A}} D_{n}^{*} \varepsilon(t) .
\end{aligned}
$$

The optimal control problem (74) can be transformed into a LQR problem

$$
\min _{U(t)} \int_{0}^{\infty}\left[\varepsilon^{T}(t) Q \varepsilon(t)+U_{\pi}^{T}(t) R U_{\pi}(t)\right] d t,
$$

where $Q=\operatorname{diag}\left\{q_{1}, \cdots, q_{n}\right\}, R=\operatorname{diag}\left\{r_{1}, \cdots, r_{n}\right\}$. Since $\left[B, A B, \cdots, A^{n-1} B\right]$ is full row rank, system (75) is controllable. There is a positive definite matrix $P \in \mathbb{R}^{N \times N}$, satisfying ARE

$$
\begin{aligned}
& \left(D_{n}^{*} I_{n} \otimes A D_{n}^{*}\right)^{T} P+P\left(D_{n}^{*} I_{n} \otimes A D_{n}^{*}\right)+Q \\
& \quad=P\left(D_{n}^{*} I_{n} \otimes B D_{n}^{*}\right) R^{-1}\left(D_{n}^{*} I_{n} \otimes B D_{n}^{*}\right)^{T} P .
\end{aligned}
$$

The optimal feedback gain matrix $D_{n}^{*} L_{f f}^{\mathcal{A}^{*}} D_{n}^{*}=R^{-1} \times\left(D_{n}^{*} I_{n} \otimes\right.$ $\left.B D_{n}^{*}\right)^{T} P$ satisfies system (75). Since $P$ is a solution of (77), the cost function is only related to $\varepsilon(t)$. Hence, the optimal topology is a star structure which enables the system to achieve the bipartite consensus.

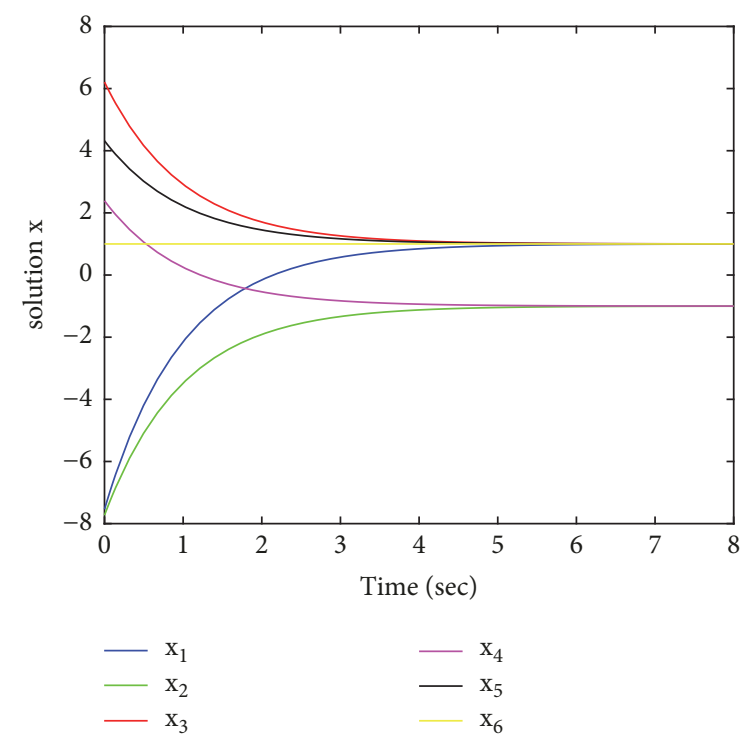

FIgURE 7: The first-order state response of MASs.

Remark 9. Theorem 8 points out that hybrid weighted star (composite) structure is the optimal topology for the firstorder and second-order system to achieve the bipartite consensus. In this situation, followers only exchange necessary information with leaders and the information exchange between followers increases the cost of control. For example, in an aircraft queue, followers are only controlled by the pilot, and no communication is performed with followers. The control cost can be effectively reduced and the target requirement can be achieved.

\section{Simulation Result}

In this section, simulations are given to show that, for the first- and second-order multiagent system, the information exchange among followers is not conducive to reducing the cost of control. Moreover, it is verified that star topology is the optimal structure for minimizing the control cost.

6.1. First-Order Multiagent System Simulation. For a firstorder multiagent system which is shown in Figures 8, 9, 10, and 11, respectively, the single leader is represented by the yellow node and the five followers are represented by nodes with other colors. The information communications between the leader and followers are hybrid-weighted. Three different topology structures are considered which are depicted by Figures 8,9 , and 10, respectively. The control cost under these topologies is listed in Table 1 with respect to different initial values. The data analysis in Table 1 shows that the control cost is minimum under star topology, and accordingly star topology is a feasible solution to achieve the optimal control. Take $Q=R=I_{5}$, and 1 as the initial state of the leader. Let $x_{0}=[-7.54,-7.73,6.21,2.39,4.32]^{T}$ be the initial state vector of followers. Then the convergence state of the firstorder MAS is shown in Figure 7. The $x$-axis and the $y$-axis in Figure 12 represent the weight variables $x$ and $y$ in Figure 11, respectively. The $z$-axis in Figure 12 represents the cost. The 
TABLE 1

\begin{tabular}{lccc}
\hline initial-value $(\mathrm{X}(0))$ & $\mathrm{J}(\mathrm{U}(\mathrm{t}), \mathrm{X}(0))_{\mathrm{G}_{\mathrm{A}}}$ & $\mathrm{J}(\mathrm{U}(\mathrm{t}), \mathrm{X}(0))_{\mathrm{G}_{\mathrm{B}}}$ & $\mathrm{J}(\mathrm{U}(\mathrm{t}), \mathrm{X}(0))_{\mathrm{G}_{\mathrm{C}}}$ \\
\hline$[3.32,6.54,-7.66,-2.78,1.77]^{T}$ & 204.75 & 185.59 & 125.95 \\
{$[4.31,4.49,-1.77,0.67,1.34]^{T}$} & 45.06 & 47.81 & 33.71 \\
{$[9.85,-8.77,-6.52,6.68,0.96]^{T}$} & 373.05 & 409.19 & 266.43 \\
{$[-7.54,-7.73,6.21,2.39,4.32]^{T}$} & 252.64 & 273.40 & 189.25 \\
{$[7.01,-7.09,8.32,-23.01,5.00]^{T}$} & 1140.20 & 1165.20 & 747.64 \\
\hline
\end{tabular}

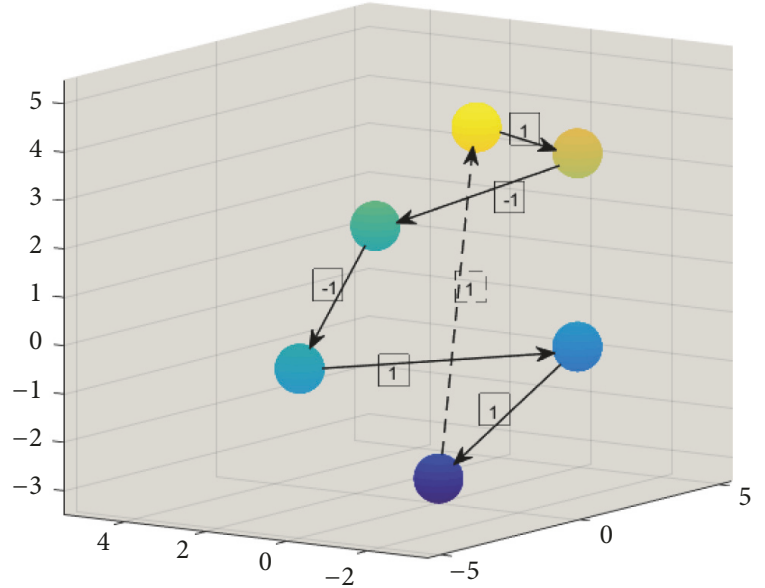

FIgURE 8

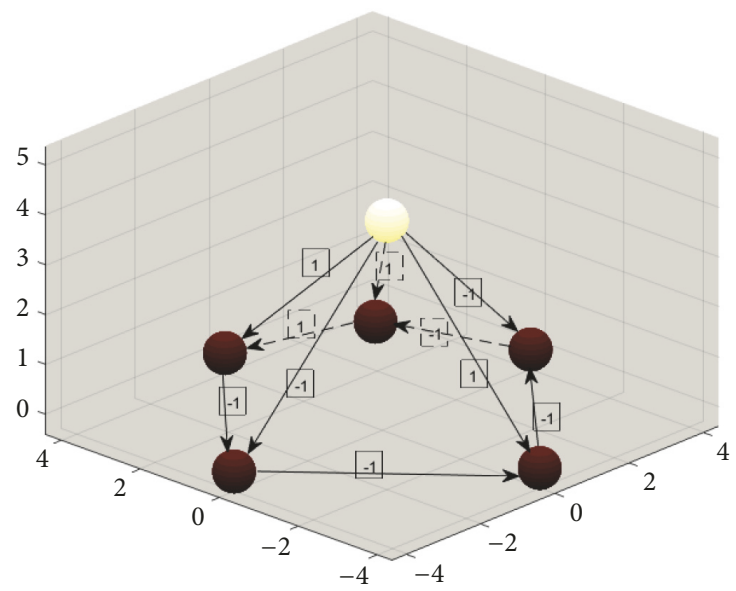

Figure 9

changes in the value of $z$-axis indicate that the control cost is lowest when the values of $x$ and $y$ are both taken as 1 . This is consistent with Theorem 2 .

6.2. Second-Order Multiagent System Simulation. For a second-order multiagent system, there are two graphs associated with state and velocity, respectively, in which the leader is represented by a yellow node and the five followers are represented with nodes of other colors. These two graphs have the same topology structure, while the edges are different. For the second-order system, the graphs associated with states are depicted by Figures 8, 9, and 10.

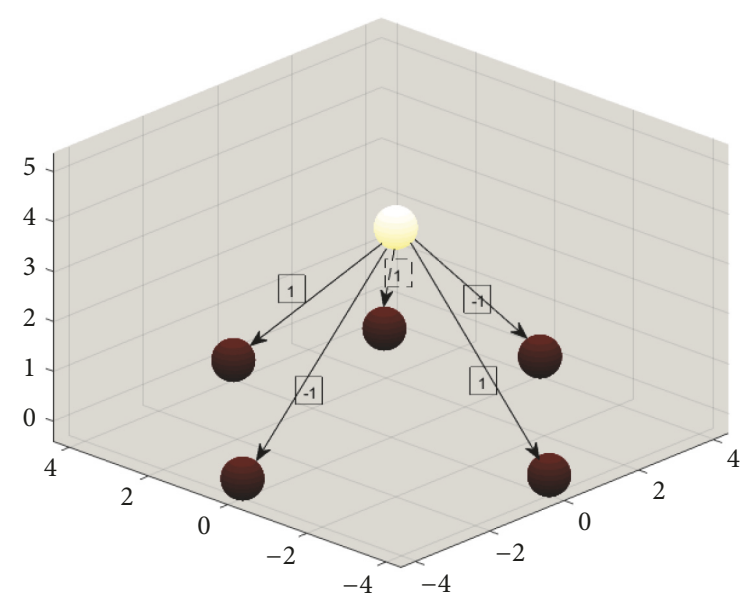

FIGURE 10

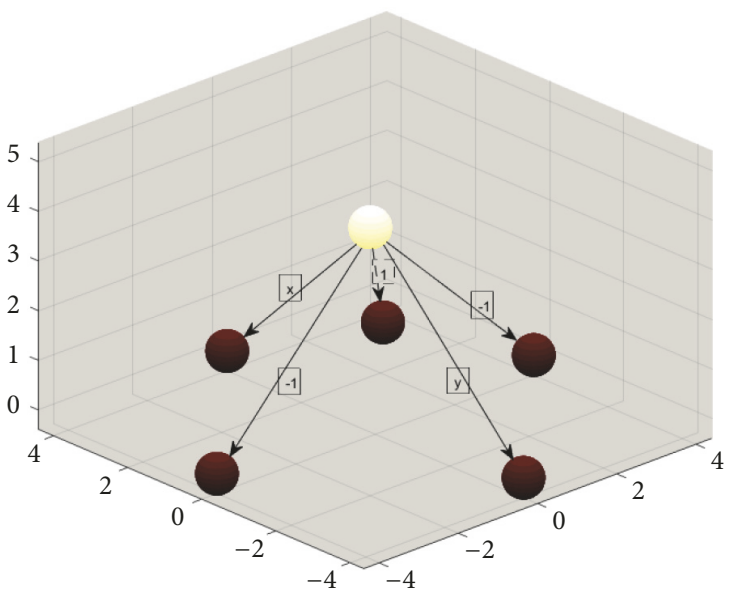

FIGURE 11

The topology structure of velocity graph is the same as state graph, while its link weights are $\sqrt{3}$ times the weights of state graph. Take $Q=R=I_{5}$, and set 0 as the initial value of state and velocity. Table 2 shows the control cost for three different topology structures when followers take different state and velocity initial values. The data analysis in Table 2 tells us that there is a minimum of control cost in the unevenly hybrid-weighted star topology. That is to say, star topology is the solution of achieving the optimal control. The initial values of followers and the leader are taken, as $\left[X_{0}, V_{0}\right]^{T}=$ $[3.77,4.21,-7.86,2.91,-1.74,6.22,3.78,-1.95,0.03,9.56]^{T}$ and 1 , respectively. Then the convergence states of MASs are 


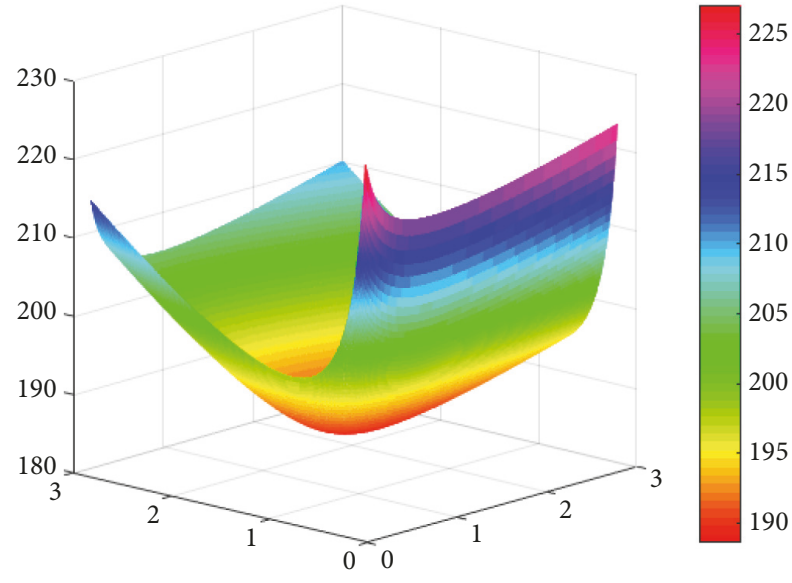

FIGURE 12

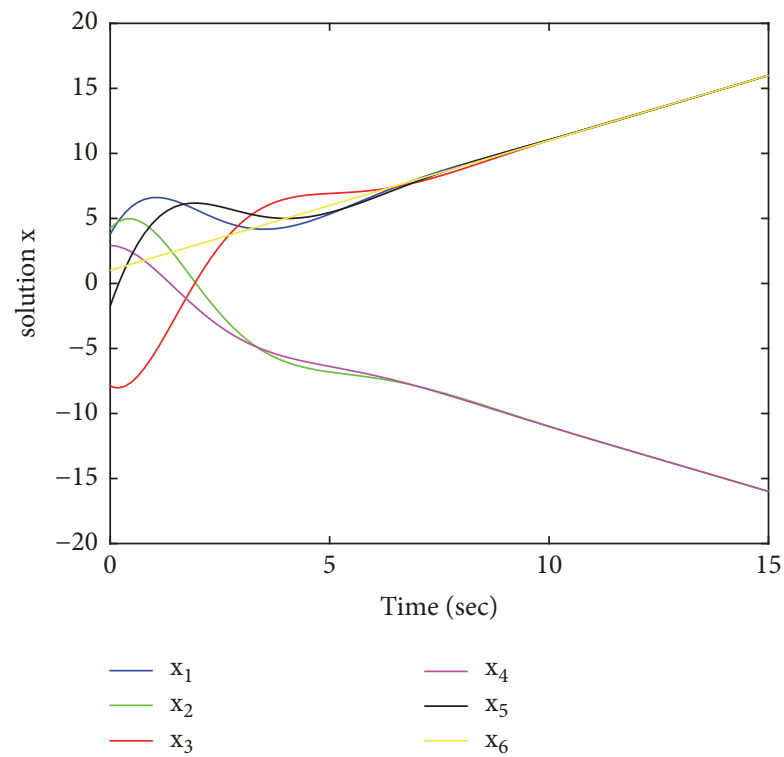

FIgURE 13: The second-order state response of MASs.

shown in Figures 13 and 14. For Figure 17, $z$-axis represents control cost. The link weights $x$ and $y$ in Figures 15 and 16 correspond to the values of $x$-axis and $y$-axis in Figure 17, respectively. By analyzing the $z$ variable in Figure 17, it can be seen that, at point $(1, \sqrt{3})$, the control cost is lowest, which conforms to Theorem 5 .

\section{Conclusion}

In this paper, we focused on the LQR based optimal control structure for multiagent systems in which the communication between agents is represented in a hybrid weighted form. We showed that the optimal topological structure is characterized by the hybrid-weighted composite star structure (the star structure is regarded as a simple composite structure). More specifically, the paper proposed a quadratic cost function independent of the topological structure first. Then, gauge transformation was implemented to the
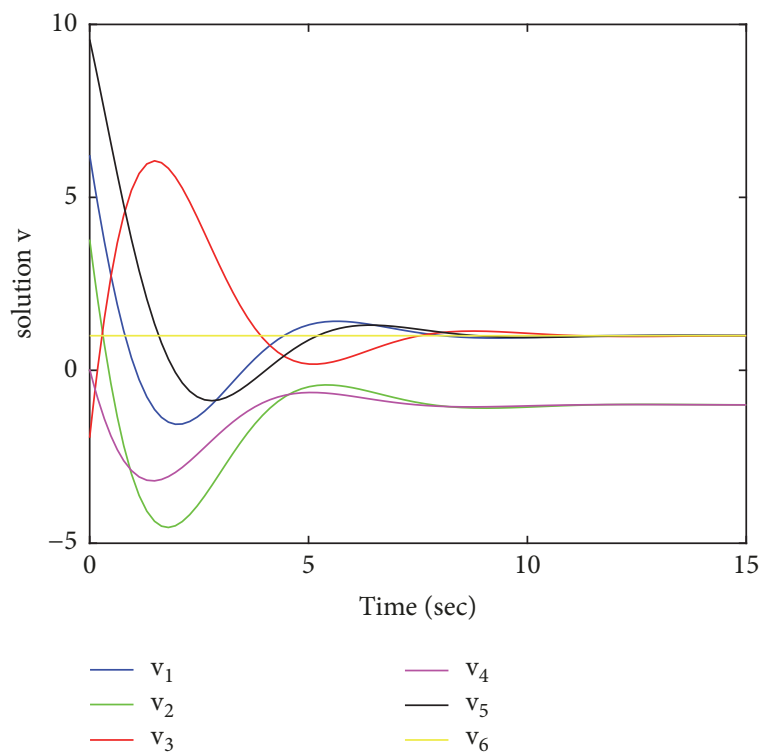

FIGURE 14: The second-order velocity response of MASs.

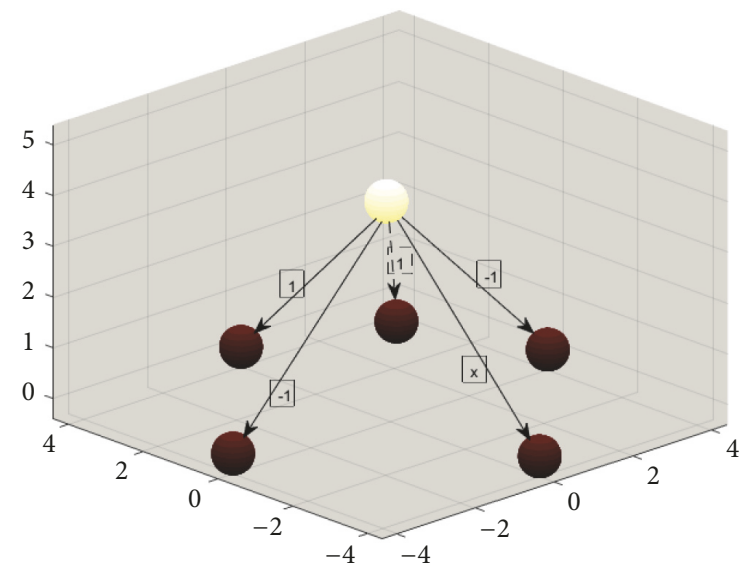

Figure 15

hybrid-weighted multiagent system. The related Riccati equation is employed for the system to obtain the optimal topology. For the first- and second-order leader-follower multiagent system, it was shown that the optimal solution always corresponds to a star composite structure, in which there is no information exchange between followers, and only leaders' communication information is received by followers. Future research will focus on the optimal control of distributed multiagent systems, such as the optimal topology of static and dynamic systems, and the convergence rate.

\section{Data Availability}

The data used to support the findings of this study are available from the corresponding author upon request.

\section{Conflicts of Interest}

The authors declare that they have no conflicts of interest. 
TABLE 2

\begin{tabular}{|c|c|c|c|}
\hline \multirow{2}{*}{ initial-value $\left([\mathrm{X}(0), \mathrm{V}(0)]^{T}\right)$} & \multicolumn{3}{|c|}{$\mathrm{J}\left(\mathrm{U}(\mathrm{t}),[\mathrm{X}(0), \mathrm{V}(0)]^{T}\right)$} \\
\hline & $G_{A}^{*}$ & $G_{B}^{*}$ & $G_{C}^{*}$ \\
\hline$[3.77,4.21,-7.86,2.91,-1.74,6.22,3.78,-1.95,0.03,9.56]^{T}$ & 715.49 & 583.15 & 439.03 \\
\hline$[22.51,51.34,-17.28,-19.74,7.39,-8.42,19.66,23.37,-28.83,43.31]^{T}$ & 22733 & 21443 & 15284 \\
\hline$[-29.31,28.41,5.21,6.79,-73.71,-80.94,91.91,-3.31,6.97,69.1]^{T}$ & 69636 & 73254 & 46528 \\
\hline$[3.33,56.8,-70.53,-9.69,58.88,6.13,-31.31,53.31,49.11,-7.11]^{T}$ & 35130 & 19349 & 17948 \\
\hline$[6.66,2.19,53.22,-7.07,0.07,88.28,67.48,-7.71,91.19,45.56]^{T}$ & 106990 & 46804 & 42090 \\
\hline
\end{tabular}

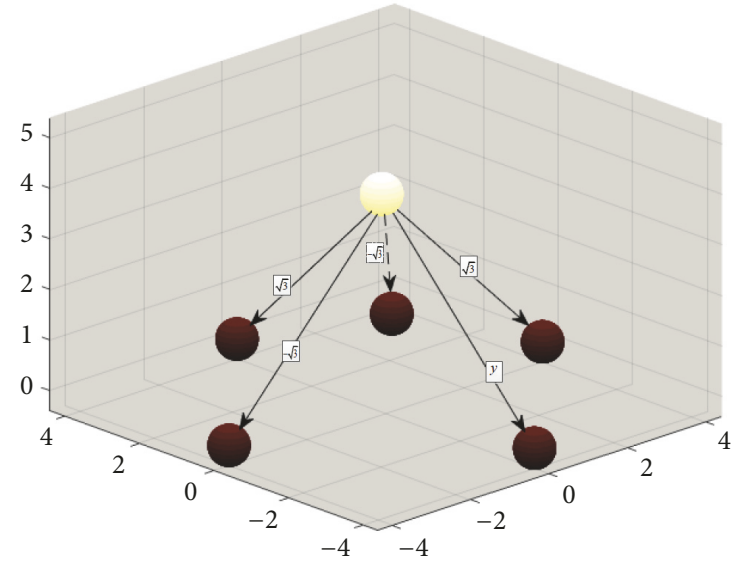

FIGURE 16

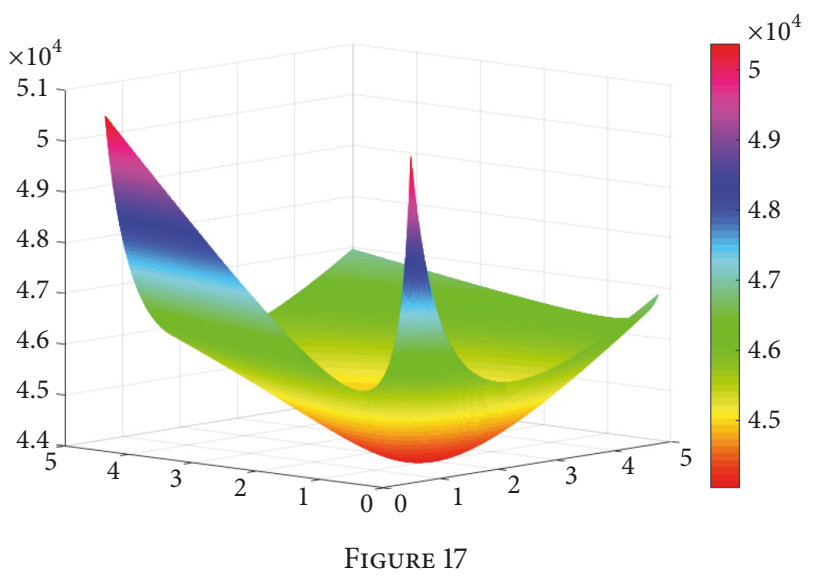

\section{Acknowledgments}

This work was supported by the National Natural Science Foundation of China under Grant 61873136, Grant 61374062, Grant 61673013, Grant 61603288, and Grant 61573203 and by the Natural Science Foundation of Shandong Province for Distinguished Young Scholars under Grant JQ201419 and Natural Science Foundation of Shandong Province under Grant ZR2015FM023 and Grant ZR2016JL022.

\section{References}

[1] L. Xiang, J. J. H. Zhu, F. Chen, and G. Chen, "Controllability of weighted and directed networks with nonidentical node dynamics," Mathematical Problems in Engineering, vol. 2013, Article ID 405034, 10 pages, 2013.

[2] Z. Ji, H. Lin, and H. Yu, "Protocols design and uncontrollable topologies construction for multi-agent networks," IEEE Transactions on Automatic Control, vol. 60, no. 3, pp. 781-786, 2015.

[3] H. Liu and H. Yu, "Decentralized state estimation for a largescale spatially interconnected system," ISA Transactions ${ }^{\circledR}$, vol. 74, pp. 67-76, 2018.

[4] J. Ma, Y. Zheng, and L. Wang, "Nash Equilibrium Topology of Multi-Agent Systems with Competitive Groups," IEEE Transactions on Industrial Electronics, vol. 64, no. 6, pp. 4956-4966, 2017.

[5] K. Liu, Z. Ji, and W. Ren, "Necessary and Sufficient Conditions for Consensus of Second-Order Multiagent Systems under Directed Topologies Without Global Gain Dependency," IEEE Transactions on Cybernetics, vol. 47, no. 8, pp. 2089-2098, 2017.

[6] H. Liu and D. Wang, "Robust state estimation for wireless sensor networks with data-driven communication," International Journal of Robust and Nonlinear Control, vol. 27, no. 18, pp. 46224632, 2017.

[7] X. Liu and Z. Ji, "Controllability of multiagent systems based on path and cycle graphs," International Journal of Robust and Nonlinear Control, vol. 28, no. 1, pp. 296-309, 2018.

[8] J. Xi, M. He, H. Liu, and J. Zheng, "Admissible output consensualization control for singular multi-agent systems with time delays," Journal of The Franklin Institute, vol. 353, no. 16, pp. 4074-4090, 2016.

[9] N. Cai, M. He, Q. Wu, and M. Khan, "On almost controllability of dynamical complex networks with noises," Journal of Systems Science and Complexity, 2018.

[10] L. Tian, Z. Ji, T. Hou, and K. Liu, "Bipartite Consensus on Coopetition Networks with Time-Varying Delays," IEEE Access, vol. 6, pp. 10169-10178, 2018.

[11] Y. Zheng, J. Ma, and L. Wang, "Consensus of hybrid multi-agent systems," IEEE Transactions on Neural Networks and Learning Systems, vol. 29, no. 4, pp. 1359-1365, 2018.

[12] Z. Ji and H. Yu, "A new perspective to graphical characterizition of multi-agent controllability," IEEE Transactions on Cybernetics, vol. 47, no. 6, pp. 1471-1483, 2017.

[13] K. Liu and Z. Ji, "Consensus of multi-agent systems with time delay based on periodic sample and event hybrid control," Neurocomputing, vol. 270, pp. 11-17, 2017.

[14] Y. Chao and Z. Ji, "Necessary and sufficient conditions for multiagent controllability of path and star topologies by exploring the information of second-order neighbours," IMA Journal of Mathematical Control \& Information, 2016.

[15] Y. Guan, Z. Ji, L. Zhang, and L. Wang, "Controllability of multiagent systems under directed topology," International Journal of Robust and Nonlinear Control, vol. 27, no. 18, pp. 4333-4347, 2017. 
[16] Y. Guan, Z. Ji, L. Zhang, and L. Wang, "Decentralized stabilizability of multi-agent systems under fixed and switching topologies," Systems \& Control Letters, vol. 62, no. 5, pp. 438446, 2013.

[17] K. Liu, Z. Ji, G. Xie, and L. Wang, "Consensus for heterogeneous multi-agent systems under fixed and switching topologies," Journal of The Franklin Institute, vol. 352, no. 9, pp. 3670-3683, 2015.

[18] Z. Lu, L. Zhang, Z. Ji, and L. Wang, "Controllability of discretetime multi-agent systems with directed topology and input delay," International Journal of Control, vol. 89, no. 1, pp. 179192, 2016.

[19] J. Ma, Y. Zheng, and L. Wang, “Topology selection for multiagent systems with opposite leaders," Systems \& Control Letters, vol. 93, pp. 43-49, 2016.

[20] F. Xiao, Y. Shi, and W. Ren, "Robustness analysis of asynchronous sampled-data multiagent networks with time-varying delays," Institute of Electrical and Electronics Engineers Transactions on Automatic Control, vol. 63, no. 7, pp. 2145-2152, 2018.

[21] Y. Guan, Z. Ji, L. Zhang, and L. Wang, "Controllability of heterogeneous multi-agent systems under directed and weighted topology," International Journal of Control, vol. 89, no. 5, pp. 1009-1024, 2016.

[22] J. Xi, Z. Fan, H. Liu, and T. Zheng, "Guaranteed-cost consensus for multiagent networks with Lipschitz nonlinear dynamics and switching topologies," International Journal of Robust and Nonlinear Control, vol. 28, no. 7, pp. 2841-2852, 2018.

[23] N. Cai, C. Diao, and M. J. Khan, "A Novel Clustering Method Based on Quasi-Consensus Motions of Dynamical Multiagent Systems," Complexity, vol. 2017, Article ID 4978613, 8 pages, 2017.

[24] Y. Zheng and L. Wang, "Consensus of switched multiagent systems," International Journal of Control, vol. 90, no. 5, pp. 11131122, 2014.

[25] Z. Lu, L. Zhang, and L. Wang, "Observability of multi-agent systems with switching topology," IEEE Transactions on Circuits and Systems II: Express Briefs, vol. 64, no. 11, pp. 1317-1321, 2017.

[26] F. Xiao, T. Chen, and H. Gao, "Consensus in time-delayed multiagent systems with quantized dwell times," Systems \& Control Letters, vol. 104, pp. 59-65, 2017.

[27] Y.-L. Wang, C.-C. Lim, and P. Shi, "Adaptively adjusted eventtriggering mechanism on fault detection for networked control systems," IEEE Transactions on Cybernetics, vol. 47, no. 8, pp. 2299-2311, 2017.

[28] J. Ma, Y. Zheng, B. Wu, and L. Wang, "Equilibrium topology of multi-agent systems with two leaders," Automatica, vol. 73, pp. 200-206, 2016.

[29] Y.-L. Wang, P. Shi, C.-C. Lim, and Y. Liu, "Event-Triggered Fault Detection Filter Design for a Continuous-Time Networked Control System," IEEE Transactions on Cybernetics, vol. 46, no. 12, pp. 3414-3426, 2016.

[30] Y. Zheng and L. Wang, "Distributed consensus of heterogeneous multi-agent systems with fixed and switching topologies," International Journal of Control, vol. 85, no. 12, pp. 1967-1976, 2012.

[31] J. Ma, Y. Zheng, and L. Wang, "Optimal topology selection for leader-following multi-agent systems with opposite leaders," in Proceedings of the 34th Chinese Control Conference, pp. 73447349, Hangzhou, China, 2015.

[32] X. Liu, Z. Ji, and T. Hou, "Graph partitions and the controllability of directed signed networks," in Science China: Information Sciences, 2018.
[33] T. Vicsek, A. Czirók, E. Ben-Jacob, I. Cohen, and O. Shochet, "Novel type of phase transition in a system of self-driven particles," Physical Review Letters, vol. 75, no. 6, pp. 1226-1229, 1995.

[34] C. Altafini, "Consensus problems on networks with antagonistic interactions," IEEE Transactions on Automatic Control, vol. 58, no. 4, pp. 935-946, 2013.

[35] J. Hu and W. Zheng, "Bipartite consensus for multi-agent systems on directed signed networks," in Proceedings of the 52nd IEEE Conference on Decision and Control (CDC '13), pp. 34513456, Florence, Italy, 2013.

[36] J. Hu and W. X. Zheng, "Emergent collective behaviors on coopetition networks," Physics Letters A, vol. 378, no. 26-27, pp. 1787-1796, 2014.

[37] Y. Cao and W. Ren, "Optimal linear-consensus algorithms: An LQR perspective," IEEE Transactions on Systems, Man, and Cybernetics, Part B: Cybernetics, vol. 40, no. 3, pp. 819-830, 2010.

[38] J. Ma, Y. Zheng, and L. Wang, "LQR-based optimal topology of leader-following consensus," International Journal of Robust and Nonlinear Control, vol. 25, no. 17, pp. 3404-3421, 2015.

[39] B. D. O. Anderson and J. B. Moore, Optimal Control: Linear Quadratic Methods, Prentice-Hall, Inc., 1990.

[40] D. Cartwright and F. Harary, "Structural balance: A generalization of heiders theory," Psychological Review, vol. 63, pp. 277292, 1956.

[41] H. Liu, G. Xie, and L. Wang, "Necessary and sufficient conditions for containment control of networked multi-agent systems," Automatica, vol. 48, no. 7, pp. 1415-1422, 2012. 


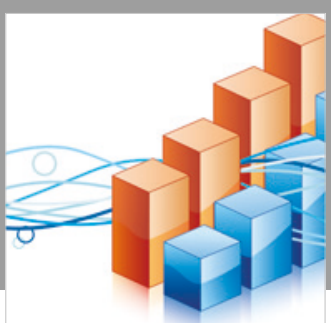

Advances in

Operations Research

\section{-n-m}
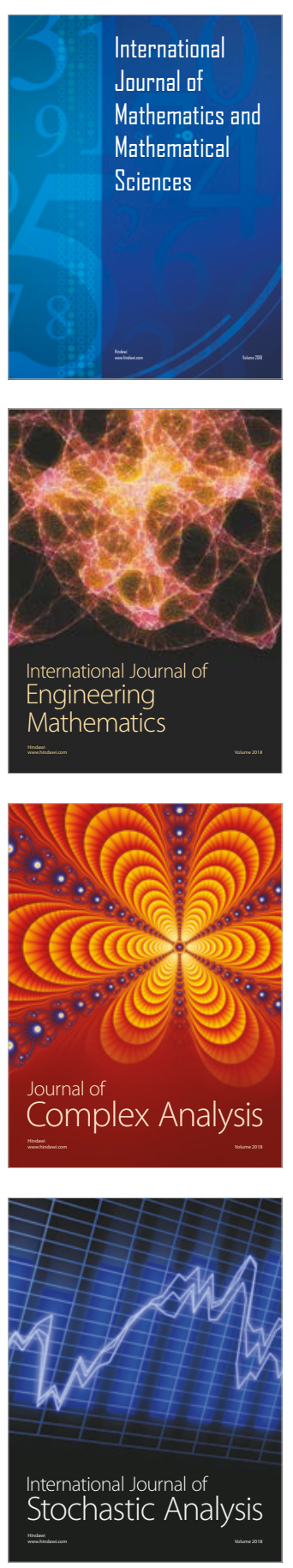
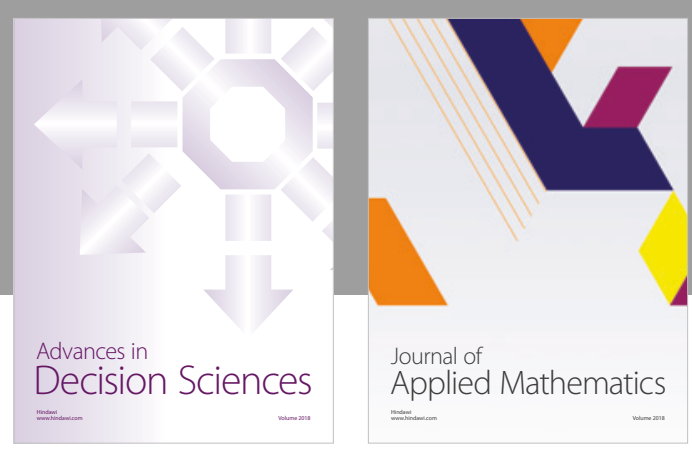

Journal of

Applied Mathematics
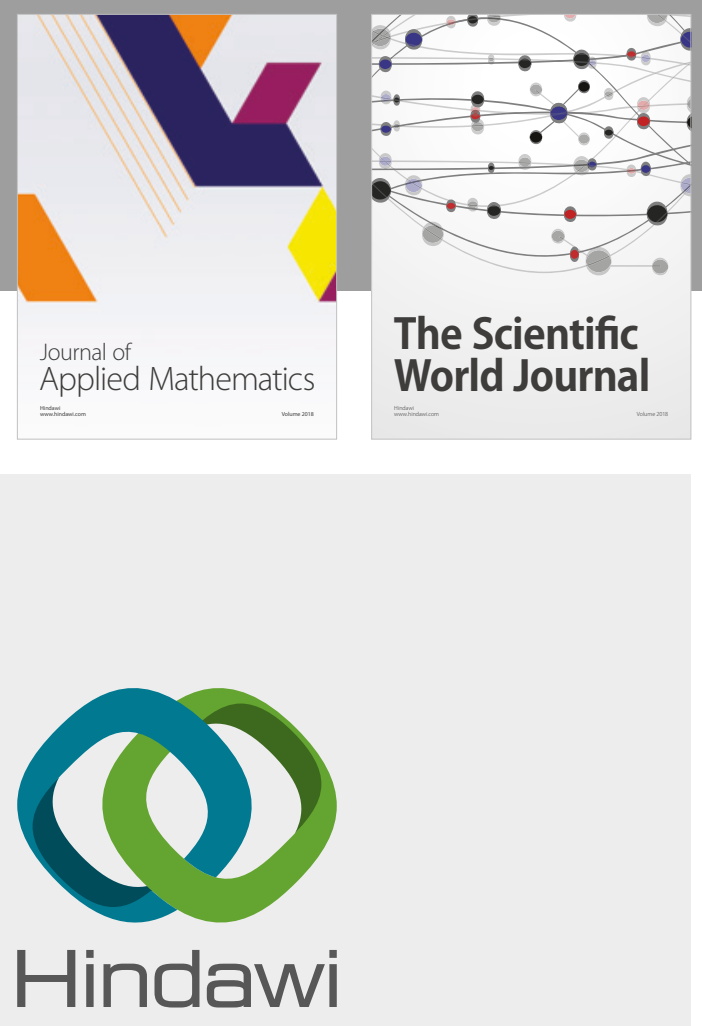

Submit your manuscripts at

www.hindawi.com

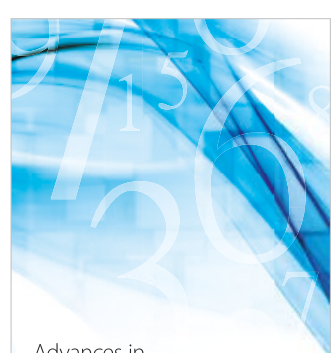

Advances in
Numerical Analysis
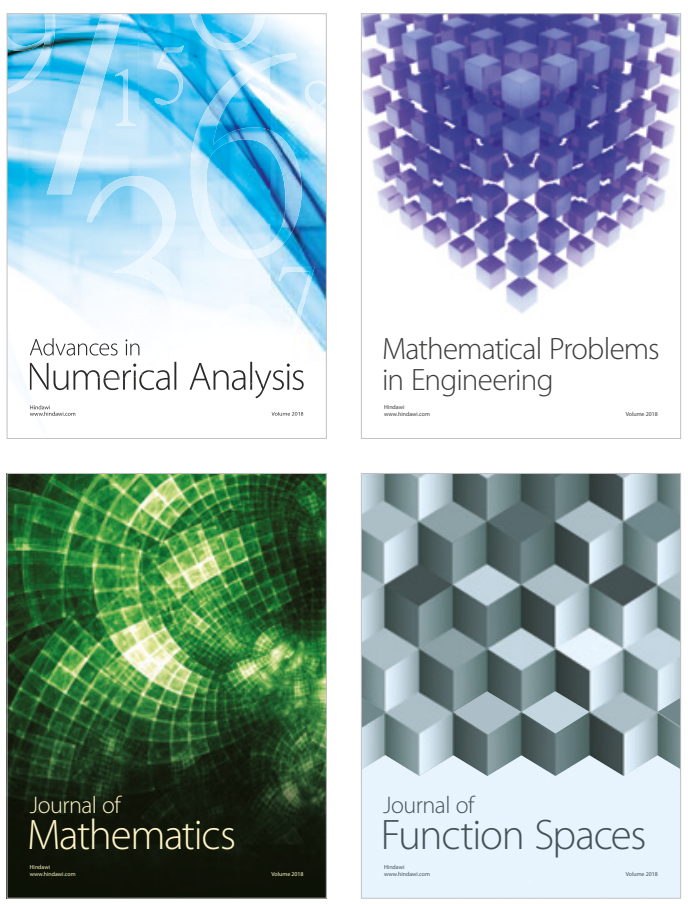

Mathematical Problems in Engineering

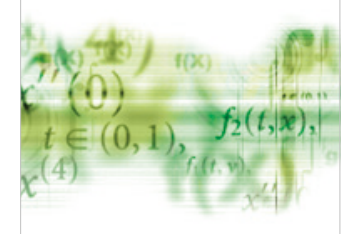

International Journal of

Differential Equations

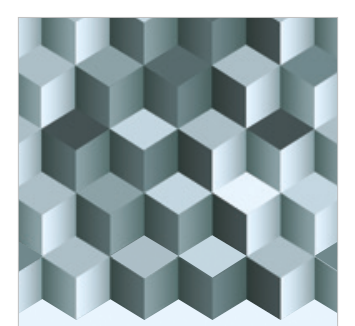

Journal of

Function Spaces
The Scientific

World Journal

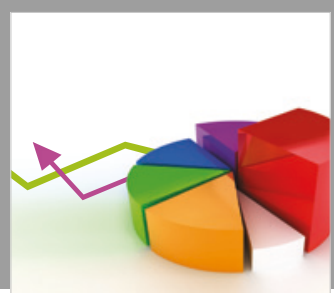

Journal of

Probability and Statistics
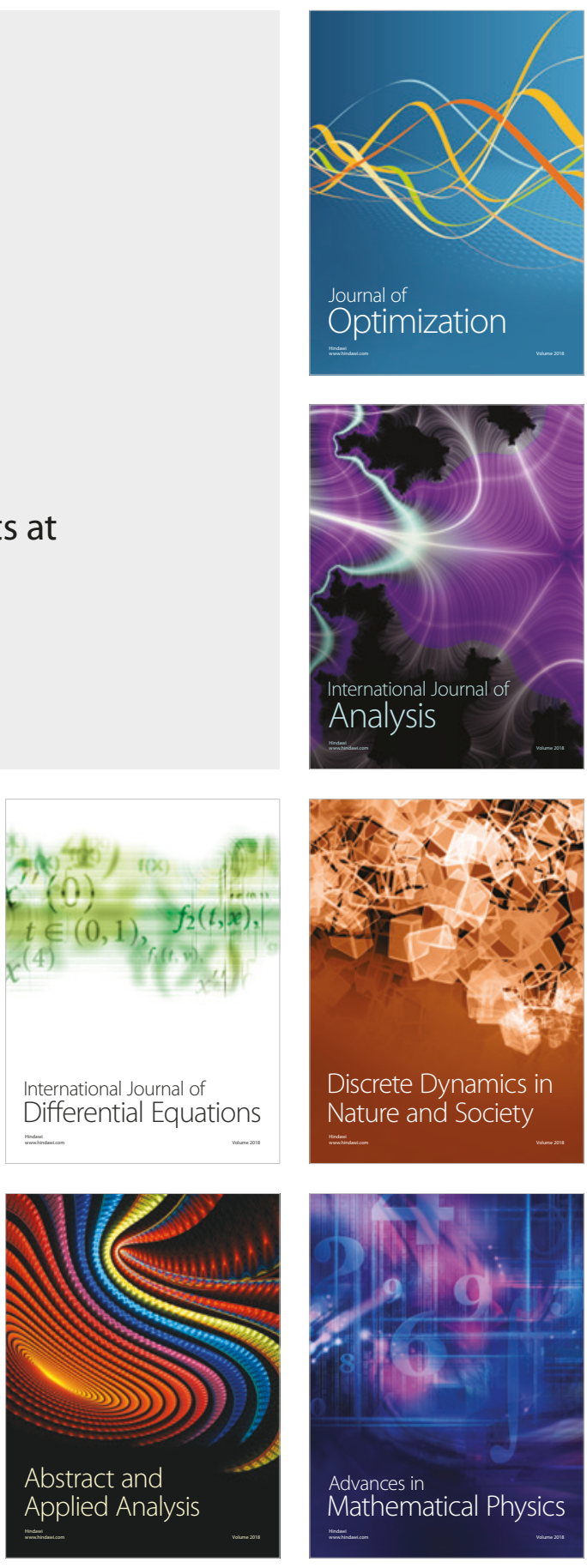